\title{
Numerical Analysis of the Fractional-Order Telegraph Equations
}

\author{
Omar Fouad Azhar, ${ }^{1}$ Muhammad Naeem $\mathbb{D}^{1},{ }^{1}$ Fatemah Mofarreh $\mathbb{D}^{2},{ }^{2}$ and Jeevan Kafle $\mathbb{D D}^{3}$ \\ ${ }^{1}$ Deanship of Joint First Year, Umm Al-Qura University, Makkah, Saudi Arabia \\ ${ }^{2}$ Department of Mathematical Sciences, College of Sciences, Princess Nourah Bint Abdulrahman University, \\ Riyadh 11546, Saudi Arabia \\ ${ }^{3}$ Central Department of Mathematics, Tribhuvan University, Kirtipur, Kathmandu, Nepal
}

Correspondence should be addressed to Jeevan Kafle; jeevan.kafle@cdmath.tu.edu.np

Received 14 July 2021; Accepted 26 July 2021; Published 10 August 2021

Academic Editor: Nehad Ali Shah

Copyright (c) 2021 Omar Fouad Azhar et al. This is an open access article distributed under the Creative Commons Attribution License, which permits unrestricted use, distribution, and reproduction in any medium, provided the original work is properly cited.

\begin{abstract}
This paper studied the fractional-order telegraph equations via the natural transform decomposition method with nonsingular kernel derivatives. The fractional result considered in the Caputo-Fabrizio derivative is Caputo sense. Currently, the communication system plays a vital role in a global society. High-frequency telecommunications continuously receive significant attention in the industry due to a slew of radiofrequency and microwave communication networks. These technologies use transmission media to move information-carrying signals from one location to another. We used natural transformation on fractional telegraph equations followed by inverse natural transformation to achieve the solution of the equation. To validate the technique, we have considered a few problems and compared them with the exact solutions.
\end{abstract}

\section{Introduction}

The telegraph equation is usually applied in signal analysis for electrical signal propagation and transmission reactiondiffusion modeling in several areas of science. It is also employed in the random one-dimensional movement of bugs along a hedge. The communication system plays a vital part in civilization around the world in the current modern era. A wide range of microwave and radiofrequency communication systems continue to benefit from significant industry attention. These technologies use media of communication to convey the signal from one place to another $[1,2]$.

These media can be divided into two classes, namely, guided and unguided. The signal is sent by the coaxial cable or a transmission line in the controlled medium. The controlled medium can carry high-frequency and current waves, whereas electromagnetic waves in unguided media transmit a signal via radiofrequency and microwave channels, part or whole communication path. An antenna is used to send and receive electromagnetic waves. The challenge of efficient telegraphic transmission is addressed with guided transmission media, notably cable transmission media. A cable com- munication channel is a directed transmission system that depicts a physical system that directly transmits data between two or more sites. To maximize the guided communication system, power and signal losses must be determined or projected, as these losses exist in all scenarios. To quantify these losses and eventually secure maximum output, some equations that can compute these losses must be developed. In practice, these equations appear in the fractional order rather than the integer order [3].

Many authors applied different analytical and numerical methods to solve the telegraph equations, such as Laplace transformation and the homotopy perturbation technique [4]. The q-homotopy analysis transformation technique is applied for the analytical result of the time-fractional telegraph equations. The reproducing kernel technique [5] and variational iteration technique are used to find the solution of the telegraph equations. The approximate solutions provided by q-HAM show convergence toward the actual result of the models [6], and the Adomian technique [7] and differential transformation technique are utilized in the analysis of the fractional-order hyperbolic telegraph equations, respectively. The solutions of the variational iteration technique 
are precisely the same as those of the Adomian decomposition technique, but the variational iteration technique required less computation [8]. The Haar wavelet [9], the generalized differential transform technique [10], the Legendre spectral Galerkin technique [11], and the linear hyperbolic telegraph equations have been solved using Sinc collocation techniques, and these methods have an exponential rate of convergence, making them particularly helpful for approximate partial differential equation solutions [12].

This article is aimed at applying the natural decomposition method (NDM) to solve telegraph equations. Rawashdeh and Maitama [13], for the first time, use natural transformation with the decomposition method for the nonlinear partial differential equations. NDM does not require prescribed assumptions, linearization, discretization, or perturbation and prevents any roundoff error. Recently, NDM is employed in the fractional-order Fisher equation [14] and fractional-order system of Burgers' equation [15].

\section{Basic Definitions}

In this section, we reproduce the definitions of RiemannLiouville (R-L), Caputo, and Caputo-Fabrizio (CF) fractional derivatives for the benefit of the reader.

Definition 1. The R-L left-sided fractional integral operator of a function $f \in C_{v}, v \geq-1$, is given as

$$
\begin{gathered}
I^{\gamma} f(\omega)=\frac{1}{\Gamma(\gamma)} \int_{0}^{\omega}(\omega-\zeta)^{\gamma-1} f(\zeta) d \zeta, \quad \gamma>0, \omega>0, \\
I^{0} f(\omega)=f(\omega) .
\end{gathered}
$$

Definition 2. The Caputo sense fractional derivative of $f(\omega)$ is defined by

$$
D_{\omega}^{\gamma} f(\omega)=I^{m-\gamma} D^{m} f(\omega)=\frac{1}{m-\gamma} \int_{\omega}^{0}(\omega-\zeta)^{m-\gamma-1} f^{m}(\zeta) d \zeta
$$

for $m-1<\gamma \leq m, m \in N, \omega>0, f \in C_{v}^{m}$, and $v \geq-1$.

Definition 3. The CF fractional derivative of $f(\omega)$ is given by

$$
D_{\omega}^{\gamma} f(\omega)=\frac{B(\gamma)}{1-\gamma} \int_{0}^{\omega} \exp \left(\frac{-\gamma(\omega-\zeta)}{1-\gamma}\right) D(f(\zeta)) d \zeta
$$

where $0<\gamma<1$ and $B(\gamma)$ is a normalization function, where $B(0)=B(1)=1$.

Definition 4. The natural transform of $\varphi(\bar{t})$ is defined by

$$
\mathcal{N}(\varphi(\bar{t}))=\mathcal{U}(s, v)=\int_{-\infty}^{\infty} e^{-s \bar{t}} \varphi(v, \bar{t}) d \bar{t}, \quad s, v \in(-\infty, \infty) .
$$

For $\bar{t} \in(0, \infty)$, the natural transform of $\varphi(\bar{t})$ is defined by $\mathcal{N}(\varphi(\bar{t}) H(\bar{t}))=\mathscr{N}^{+}=\mathscr{U}^{+}(s, v)=\int_{-\infty}^{\infty} e^{-s \bar{t}} \varphi(v, \bar{t}) d \bar{t}, \quad s, v \in(0, \infty)$,

where $H(\bar{t})$ is the Heaviside function.

Definition 5. The inverse natural transformation of $\mathcal{U}(s, v)$ is given by

$$
\mathcal{N}^{-1}[\mathcal{U}(s, v)]=\varphi(\bar{t}), \quad \forall \bar{t} \geq 0 .
$$

Lemma 6 (Linearity property). If the natural transform of $\varphi_{1}(\bar{t})$ is $\varphi_{1}(s, v)$ and $\varphi_{2}(\bar{t})$ is $\varphi_{2}(s, v)$, then

$\mathcal{N}\left[c_{1} \varphi_{1}(\bar{t})+c_{2} \varphi_{2}(\bar{t})\right]=c_{1} \mathcal{N}\left[\varphi_{1}(\bar{t})\right]+c_{2} \mathcal{N}\left[\varphi_{2}(\bar{t})\right]=c_{1} \varphi_{1}(s, v)+c_{2} \varphi_{2}(s, v)$

where $c_{1}$ and $c_{2}$ are constants.

Lemma 7 (Inverse property). If the inverse natural transform of $\varphi_{1}(s, v)$ and $\varphi_{2}(s, v)$ is $\varphi_{1}(\bar{t})$ and $\varphi_{2}(\bar{t})$, respectively, then

$$
\begin{aligned}
\mathcal{N}^{-1}\left[c_{1} \varphi_{1}(s, v)+c_{2} \varphi_{2}(s, v)\right] & =c_{1} \mathcal{N}^{-1}\left[\varphi_{1}(s, v)\right]+c_{2} \mathcal{N}^{-1}\left[\varphi_{2}(s, v)\right] \\
& =c_{1} \varphi_{1}(\bar{t})+c_{2} \varphi_{2}(\bar{t})
\end{aligned}
$$

where $c_{1}$ and $c_{2}$ are constants.

Definition 8 . The natural transform of $D_{\bar{t}}^{\gamma} \varphi(\bar{t})$ by means of Caputo sense is given as

$$
\mathcal{N}\left[D_{\bar{t}}^{\gamma}\right]=\left(\frac{s}{v}\right)^{\gamma}\left(\mathcal{N}[\varphi(\bar{t})]-\left(\frac{1}{s}\right) \varphi(0)\right) .
$$

Definition 9. The natural transform of $D_{\bar{t}}^{\gamma} \varphi(\bar{t})$ by means of CF is defined as

$$
\mathcal{N}\left[D_{\bar{t}}^{\gamma}\right]=\frac{1}{1-\gamma+\gamma(v / s)}\left(\mathcal{N}[\varphi(\bar{t})]-\left(\frac{1}{s}\right) \varphi(0)\right)
$$

\section{Methodology}

In this section, we present a novel approximate analytical procedure based on the natural transform to the following equation:

$$
D_{\bar{t}}^{\gamma} \varphi(\bar{\xi}, \bar{t})=\mathscr{L}(\varphi(\bar{\xi}, \bar{t}))+\mathcal{N}(\varphi(\bar{\xi}, \bar{t}))+h(\bar{\xi}, \bar{t})
$$

with the initial condition

$$
\varphi(\bar{\xi}, 0)=\phi(\bar{\xi})
$$



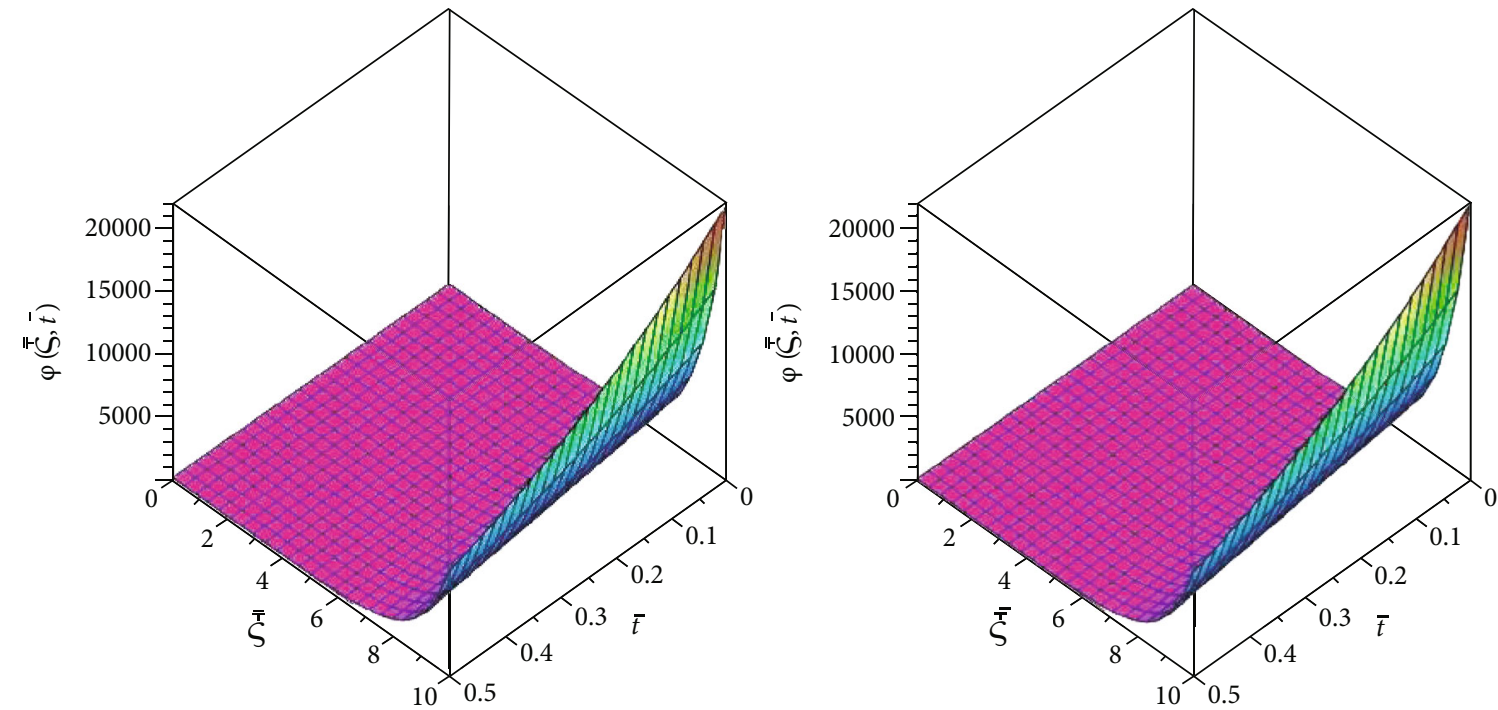

Figure 1: The exact and analytical solution figures of Example 1 at $\gamma=1$.

where $\mathcal{N}, \mathscr{L}$, and $h(\bar{\xi}, \bar{t})$ are nonlinear, linear, and source terms, respectively. Now, we employ NT to equation (11) by considering fractional derivatives using two fractional definitions.

By taking the natural transform of equation (11) by means of the $\mathrm{CF}$ fractional derivative, we obtain

$$
\frac{1}{p(\gamma, v, s)}\left(\mathcal{N}[\varphi(\bar{\xi}, \bar{t})]-\frac{\phi(\bar{\xi})}{s}\right)=\mathscr{N}[M(\bar{\xi}, \bar{t})]
$$

where

$$
p(\gamma, v, s)=1-\gamma+\gamma\left(\frac{v}{s}\right)
$$

By taking the inverse natural transform using (6), we rewrite (13) as

$$
\varphi(\bar{\xi}, \bar{t})=\mathcal{N}^{-1}\left(\frac{\phi(\bar{\xi})}{s}+p(\gamma, v, s) \mathcal{N}[M(\bar{\xi}, \bar{t})]\right) .
$$

$\mathcal{N}(\varphi(\bar{\xi}, \bar{t}))$ can be decomposed into

$$
\mathcal{N}(\varphi(\bar{\xi}, \bar{t}))=\sum_{i=0}^{\infty} A_{\bar{t}}
$$

where $A_{\bar{t}}$ is the Adomian polynomial $[2,4]$. We assume that equation (11) has the analytical expansion

$$
\varphi(\bar{\xi}, t)=\sum_{i=0}^{\infty} \varphi_{i}(\bar{\xi}, \bar{t})
$$

By substituting equations (16) and (17) into (15), we obtain

$$
\begin{aligned}
\sum_{i=0}^{\infty} \varphi_{i}(\bar{\xi}, \bar{t})= & \mathcal{N}^{-1}\left(\frac{\phi(\bar{\xi})}{s}+p(\gamma, v, s) \mathcal{N}[h(\bar{\xi}, \bar{t})]\right) \\
& +\mathcal{N}^{-1}\left(p(\gamma, v, s) \mathcal{N}\left[\sum_{i=0}^{\infty} \mathscr{L}\left(\varphi_{i}(\bar{\xi}, \bar{t})\right)+A_{\bar{t}}\right]\right)
\end{aligned}
$$

From (18), we get

$$
\begin{gathered}
\varphi_{0}^{\mathrm{CF}}(\bar{\xi}, \bar{t})=\mathcal{N}^{-1}\left(\frac{\phi(\bar{\xi})}{s}+p(\gamma, v, s) \mathcal{N}[h(\bar{\xi}, \bar{t})]\right), \\
\varphi_{1}^{\mathrm{CF}}(\bar{\xi}, \bar{t})=\mathcal{N}^{-1}\left(p(\gamma, v, s) \mathcal{N}\left[\mathscr{L}\left(\varphi_{0}(\bar{\xi}, \bar{t})\right)+A_{0}\right]\right), \\
\vdots \\
\varphi_{l+1}^{\mathrm{CF}}(\bar{\xi}, \bar{t})=\mathcal{N}^{-1}\left(p(\gamma, v, s) \mathcal{N}\left[\mathscr{L}\left(u_{l}(\bar{\xi}, \bar{t})\right)+A_{l}\right]\right), \quad l=1,2,3, \cdots
\end{gathered}
$$

By substituting (19) into (17), we get the $\mathrm{NDM}_{\mathrm{CF}}$ solution of (11) as

$$
\varphi^{\mathrm{CF}}(\bar{\xi}, \bar{t})=\varphi_{0}^{\mathrm{CF}}(\bar{\xi}, \bar{t})+\varphi_{1}^{\mathrm{CF}}(\bar{\xi}, \bar{t})+\varphi_{2}^{\mathrm{CF}}(\bar{\xi}, \bar{t})+\cdots
$$

\section{Convergence Analysis}

We have presented uniqueness and convergence of the ND $\mathrm{M}_{\mathrm{CF}}$ in this section.

Theorem 10. The NTDM $M_{C F}$ solution of (11) is unique when $0<\left(\delta_{1}+\delta_{2}\right)(1-\gamma+\gamma \bar{t})<1$. 

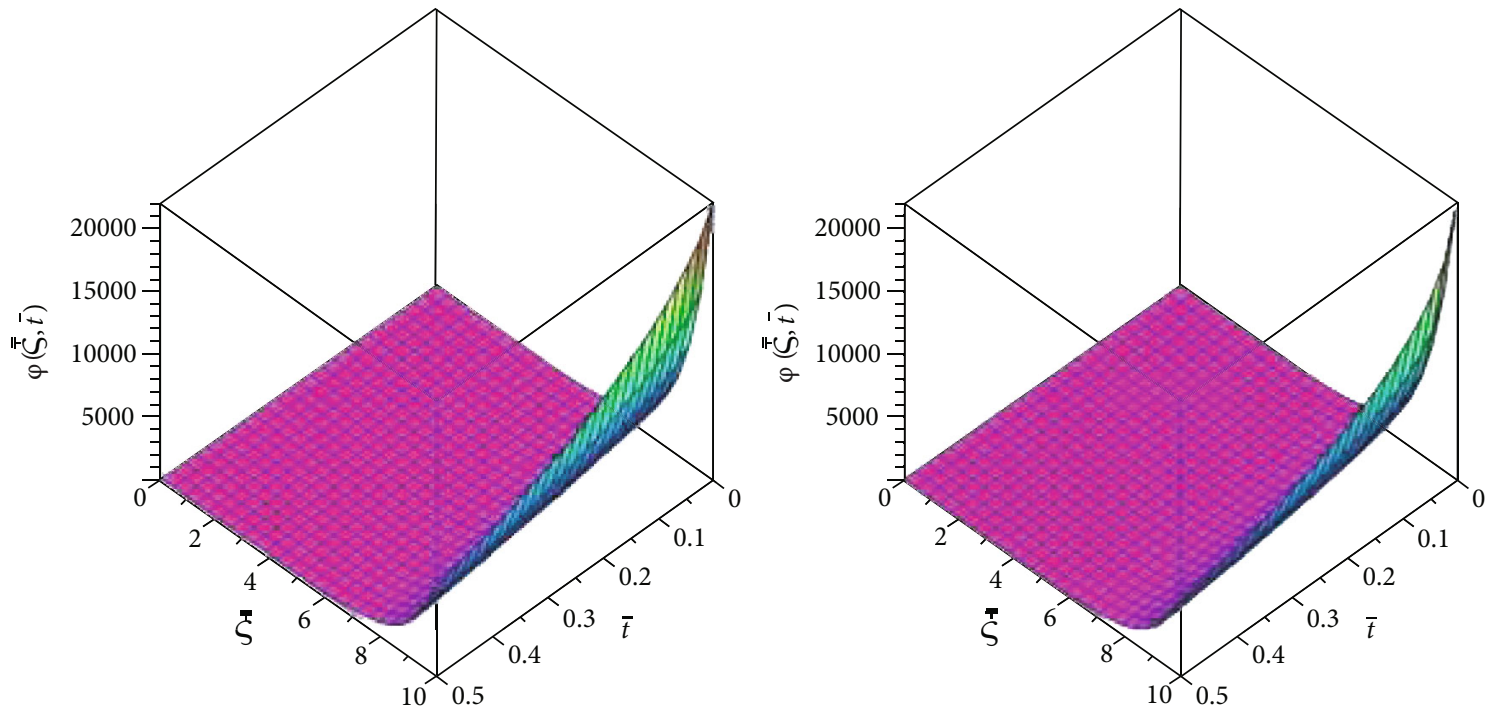

Figure 2: The different fractional-order graphs of $\varphi(\bar{\xi}, \bar{t})$ at $\gamma=0.8$ and $\gamma=0.6$.

Proof. Let $F=(C[J],\|\|$.$) be the Banach space with the norm$ $\|\phi(t)\|=\max _{t \in J}|\phi(t)|, \forall$ continuous function on $J$. Let $G$ $: F \longrightarrow F$ be a nonlinear mapping, where

$$
\varphi_{l+1}^{C}=\varphi_{0}^{C}+\mathscr{N}^{-1}\left[p(\gamma, v, s) \cdot \mathcal{N}\left[\mathscr{L}\left(\varphi_{l}(\bar{x} i, \bar{t})\right)+\mathcal{N}\left(\varphi_{l}(\bar{x} i, \bar{t})\right)\right]\right], \quad l \geq 0 .
$$

Suppose that $\left|\mathscr{L}(\varphi)-\mathscr{L}\left(\varphi^{*}\right)\right|<\delta_{1}\left|\varphi-\varphi^{*}\right|$ and $\mid \mathcal{N}(\varphi)-$ $\mathcal{N}\left(\varphi^{*}\right)\left|<\delta_{2}\right| \varphi-\varphi^{*} \mid$, where $\delta_{1}$ and $\delta_{2}$ are Lipschitz constants and $\varphi:=\varphi(\zeta, \bar{t})$ and $\varphi^{*}:=\varphi^{*}(\zeta, t)$ are two different function values.

$$
\begin{aligned}
\left\|G \varphi-G \varphi^{*}\right\| \leq & \max _{\bar{t} \in J} \mid \mathcal{N}^{-1}\left[p(\gamma, v, s) \mathcal{N}\left[\mathscr{L}(\varphi)-\mathscr{L}\left(\varphi^{*}\right)\right]\right. \\
& \left.+p(\gamma, v, s) \mathcal{N}\left[\mathcal{N}(\varphi)-\mathcal{N}\left(\varphi^{*}\right)\right] \mid\right] \\
\leq & \max _{\bar{t} \in J}\left[\delta_{1} \mathcal{N}^{-1}\left[p(\gamma, v, s) \mathscr{N}\left[\left|\varphi-\varphi^{*}\right|\right]\right]\right. \\
& \left.+\delta_{2} \mathcal{N}^{-1}\left[p(\gamma, v, s) \mathcal{N}\left[\left|\varphi-\varphi^{*}\right|\right]\right]\right] \\
\leq & \max _{\bar{t} \in J}\left(\delta_{1}+\delta_{2}\right)\left[\mathcal{N}^{-1}\left[p(\gamma, v, s) \mathcal{N}\left|\varphi-\varphi^{*}\right|\right]\right] \\
\leq & \left(\delta_{1}+\delta_{2}\right)\left[\mathcal{N}^{-1}\left[p(\gamma, v, s) \mathcal{N}\left\|\varphi-\varphi^{*}\right\|\right]\right] \\
= & \left(\delta_{1}+\delta_{2}\right)(1-\gamma+\gamma \bar{t})\left\|\varphi-\varphi^{*}\right\| .
\end{aligned}
$$

Gis a contraction of $0<\left(\delta_{1}+\delta_{2}\right)(1-\gamma+\gamma \bar{t})<1$. The solution of (11) is unique from the Banach fixed-point theorem.

Theorem 11. The $N T D M_{C F}$ solution of (11) is convergent.
Proof. Let $\varphi_{m}=\sum_{r=0}^{m} \varphi_{r}(\bar{\xi}, \bar{t})$. To prove that $\varphi_{m}$ is a Cauchy sequence in $F$. Consider

$$
\begin{aligned}
& \left\|\varphi_{m}-\varphi_{n}\right\|=\max _{\overline{t \in J}}\left|\sum_{r=n+1}^{m} \varphi_{r}\right| \\
& \leq \max _{\overline{i \epsilon} \mid}\left|\mathcal{N}^{-1}\left[p(\gamma, v, s) \mathcal{N}\left[\sum_{r=n+1}^{m}\left(\mathscr{L}\left(\varphi_{r-1}\right)+\mathcal{N}\left(\varphi_{r-1}\right)\right)\right]\right]\right| \\
& =\max _{i \epsilon \mid}\left|\mathscr{N}^{-1}\left[p(\gamma, v, s) \cdot \mathcal{N}\left[\sum_{r=n+1}^{m-1}\left(\mathscr{L}\left(\varphi_{r}\right)+\mathscr{N}\left(\varphi_{r}\right)\right)\right]\right]\right| \\
& \leq \max _{i \in\}}\left|\mathscr{N}^{-1}\left[p(\gamma, v, s) \cdot \mathcal{N}\left[\left(\mathscr{L}\left(\varphi_{m-1}\right)-\mathscr{L}\left(\varphi_{n-1}\right)+\mathcal{N}\left(\varphi_{m-1}\right)-\mathcal{N}\left(\varphi_{n-1}\right)\right)\right]\right]\right| \\
& \leq \delta_{1} \max _{i \in \in}\left|\cdot \mathcal{N}^{-1}\left[p(\gamma, v, s) \mathcal{N}\left[\left(\mathscr{L}\left(\varphi_{m-1}\right)-\mathscr{L}\left(\varphi_{n-1}\right)\right)\right]\right]\right| \\
& +\delta_{2} \max _{\epsilon \in \in}\left|\cdot \mathcal{N}^{-1}\left[p(\gamma, v, s) \cdot \mathcal{N}\left[\left(\mathcal{N}\left(u_{m-1}\right)-\mathcal{N}\left(\bar{t}_{n-1}\right)\right)\right]\right]\right| \\
& =\left(\delta_{1}+\delta_{2}\right)(1-\gamma+\gamma \bar{t})\left\|\varphi_{m-1}-\varphi_{n-1}\right\|, \quad n=1,2,3, \cdots .
\end{aligned}
$$

Let $m=n+1$; then,

$\left\|\varphi_{n+1}-\varphi_{n}\right\| \leq \delta\left\|\varphi_{n}-\varphi_{n-1}\right\| \leq \delta^{2}\left\|\varphi_{n-1} \varphi_{n-2}\right\| \leq \cdots \leq \delta^{n}\left\|\varphi_{1}-\varphi_{0}\right\|$,

where $\delta=\left(\delta_{1}+\delta_{2}\right)(1-\gamma+\gamma \bar{t})$. Similarly, we have

$$
\begin{gathered}
\left\|\varphi_{m}-\varphi_{n}\right\| \leq\left\|\varphi_{n+1}-\varphi_{n}\right\|+\left\|\varphi_{n+2} \varphi_{n+1}\right\|+\cdots+\left\|\varphi_{m}-\varphi_{m-1}\right\|, \\
\left(\delta^{n}+\delta^{n+1}+\cdots+\delta^{m-1}\right)\left\|\varphi_{1}-\varphi_{0}\right\| \leq \delta^{n}\left(\frac{1-\delta^{m-n}}{1-\delta}\right)\left\|\varphi_{1}\right\| .
\end{gathered}
$$

As $0<\delta<1$, we get $1-\delta^{m-n}<1$. Therefore,

$$
\left\|\varphi_{m}-\varphi_{n}\right\| \leq \frac{\delta^{n}}{1-\delta} \max _{\bar{t} \in J}\left\|\varphi_{1}\right\| .
$$

Since $\left\|\varphi_{1}\right\|<\infty,\left\|\varphi_{m}-\varphi_{n}\right\| \longrightarrow 0$ when $n \longrightarrow \infty$. Hence, 


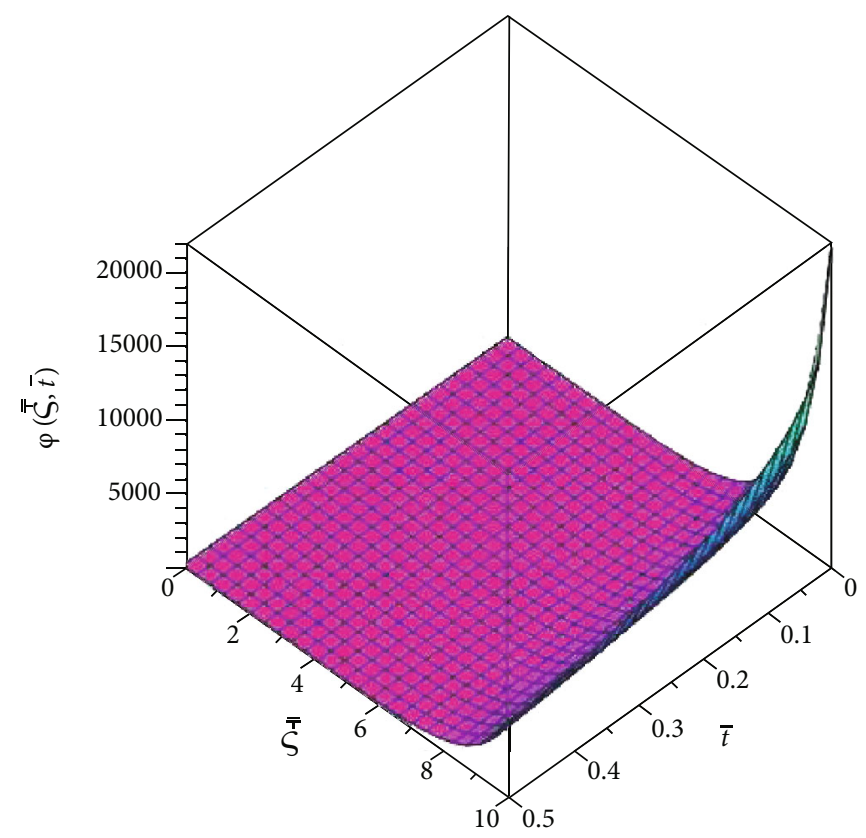

FIgURE 3: The fractional-order graph at $\gamma=0.40$.

$\varphi_{m}$ is a Cauchy sequence in $F$; therefore, the series $\varphi_{m}$ is convergent.

\section{Results}

Example 1. Consider the fractional-order telegraph equation

$$
\frac{\partial^{2 \gamma} \varphi}{\partial \bar{t}^{2 \gamma}}+2 \frac{\partial^{\gamma} \varphi}{\partial \bar{t}^{\gamma}}+\varphi=\frac{\partial^{2} \varphi}{\partial \bar{\xi}^{2}}, \quad 0<\gamma \leq 1, t \geq 0
$$

with the initial condition

$$
\begin{gathered}
\varphi(\bar{\xi}, 0)=e^{\bar{\xi}} \\
\varphi_{\bar{t}}(\bar{\xi}, 0)=-2 e^{\bar{\xi}} .
\end{gathered}
$$

Now, applying the natural transformation to equation (27), we get

$$
\mathcal{N}\left[\frac{\partial^{2 \gamma} \varphi}{\partial \bar{t}^{2 \gamma}}\right]=-\mathcal{N}\left[2 \frac{\partial^{\gamma} \varphi}{\partial \bar{t}^{\gamma}}+\varphi-\frac{\partial^{2} \varphi}{\partial \bar{\xi}^{2}}\right]
$$

Using the inverse natural transformation,

$$
\varphi(\bar{\xi}, \bar{t})=\mathscr{N}^{-1}\left[\frac{\varphi(\bar{\xi}, 0)}{s}+\frac{\mu}{s^{2}} \varphi_{\bar{t}}(\bar{\xi}, 0)-\frac{(\mu / s)+\gamma(1-(\mu / s))}{(\mu / s)^{2}} \mathcal{N}\left[2 \frac{\partial^{\gamma} \varphi}{\partial \bar{t}^{\gamma}}+\varphi-\frac{\partial^{2} \varphi}{\partial \bar{\xi}^{2}}\right]\right] .
$$

Using the ADM procedure, we get

$$
\begin{gathered}
\varphi_{0}(\bar{\xi}, \bar{t})=\mathcal{N}^{-1}\left[\frac{\varphi(\bar{\xi}, 0)}{s}+\frac{\mu}{s^{2}} \varphi_{\bar{t}}(\bar{\xi}, 0)\right]=\mathcal{N}^{-1}\left[\frac{e^{\bar{\xi}}}{s}-\frac{2 \mu e^{\bar{\xi}}}{s^{2}}\right], \\
\varphi_{0}(\bar{\xi}, \bar{t})=e^{\bar{\xi}}(1-2 \bar{t}), \\
\varphi_{j+1}=-\mathcal{N}^{-1}\left[\frac{(\mu / s)+\gamma(1-(\mu / s))}{(\mu / s)^{2}} \mathcal{N}\left[2 \frac{\partial^{\gamma} \varphi_{j}}{\partial \bar{t}^{\gamma}}+\varphi_{j}-\frac{\partial^{2} \varphi_{j}}{\partial \bar{\xi}^{2}}\right]\right], \quad j=0,1,2, \cdots
\end{gathered}
$$

For $j=0$,

$$
\begin{aligned}
\varphi_{1}(\bar{\xi}, \bar{t}) & =-\mathcal{N}^{-1}\left[\frac{(\mu / s)+\gamma(1-(\mu / s))}{(\mu / s)^{2}} \mathcal{N}\left[2 \frac{\partial^{\gamma} \varphi_{0}}{\partial \bar{t}^{\gamma}}+\varphi_{0}-\frac{\partial^{2} \varphi_{0}}{\partial \bar{\xi}^{2}}\right]\right] \\
& =2 e^{\bar{\xi}} \bar{t}(2-2 \gamma+\gamma \bar{t}) .
\end{aligned}
$$

The subsequent terms are

$$
\begin{aligned}
\varphi_{2}(\bar{\xi}, \bar{t}) & =-\mathcal{N}^{-1}\left[\frac{(\mu / s)+\gamma(1-(\mu / s))}{(\mu / s)^{2}} \mathcal{N}\left[2 \frac{\partial^{\gamma} \varphi_{1}}{\partial \bar{t}^{\gamma}}+\varphi_{1}-\frac{\partial^{2} \varphi_{1}}{\partial \bar{\xi}^{2}}\right]\right] \\
& =-\frac{4 e^{\bar{\xi}} \bar{t}^{2}}{3}(3-3 \gamma+\gamma \bar{t}), \\
\varphi_{3}(\bar{\xi}, \bar{t}) & =-\mathcal{N}^{-1}\left[\frac{(\mu / s)+\gamma(1-(\mu / s))}{(\mu / s)^{2}} \mathcal{N}\left[2 \frac{\partial^{\gamma} \varphi_{2}}{\partial \bar{t}^{\gamma}}+\varphi_{2}-\frac{\partial^{2} \varphi_{2}}{\partial \bar{\xi}^{2}}\right]\right] \\
& =\frac{2 e^{\bar{\xi}} \overline{\bar{t}}^{3}}{3}(4-4 \gamma+\gamma \bar{t}),
\end{aligned}
$$

The NDM result for Example 1 is

$$
\begin{aligned}
\varphi(\bar{\xi}, \bar{t})= & \varphi_{0}(\bar{\xi}, \bar{t})+\varphi_{1}(\bar{\xi}, \bar{t})+\varphi_{2}(\bar{\xi}, \bar{t})+\varphi_{3}(\bar{\xi}, \bar{t})+\varphi_{4}(\bar{\xi}, \bar{t}) \cdots \\
\varphi(\bar{\xi}, \bar{t})= & e^{\bar{\xi}}\left[1-2 \bar{t}+2 e^{\bar{\xi}} \bar{t}(2-2 \gamma+\gamma \bar{t})-\frac{4 e^{\bar{\xi}} \bar{t}^{2}}{3}(3-3 \gamma+\gamma \bar{t})\right. \\
& \left.+\frac{2 e^{\bar{\xi}} \bar{t}^{3}}{3}(4-4 \gamma+\gamma \bar{t})+\cdots\right] .
\end{aligned}
$$

When $\gamma=1$, then the NDM result is

$$
\varphi(\bar{\xi}, \bar{t})=e^{\bar{\xi}}\left[1-2 \bar{t}+\frac{(2 \bar{t})^{2}}{2 !}-\frac{(2 \bar{t})^{3}}{3 !}+\frac{(2 \bar{t})^{4}}{4 !} \cdots\right]
$$



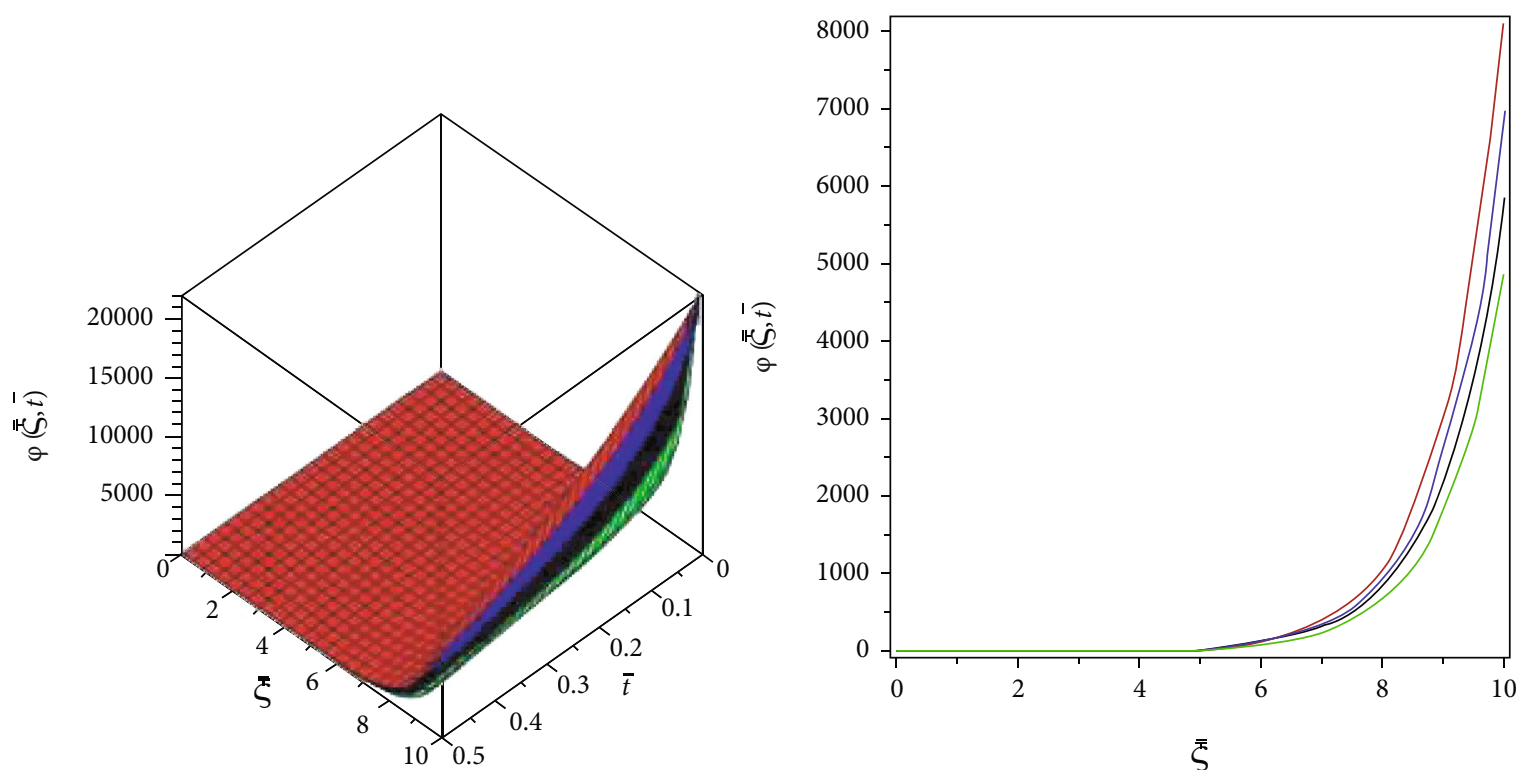

Figure 4: The 3D and 2D different fractional-order graphs of $\gamma$.
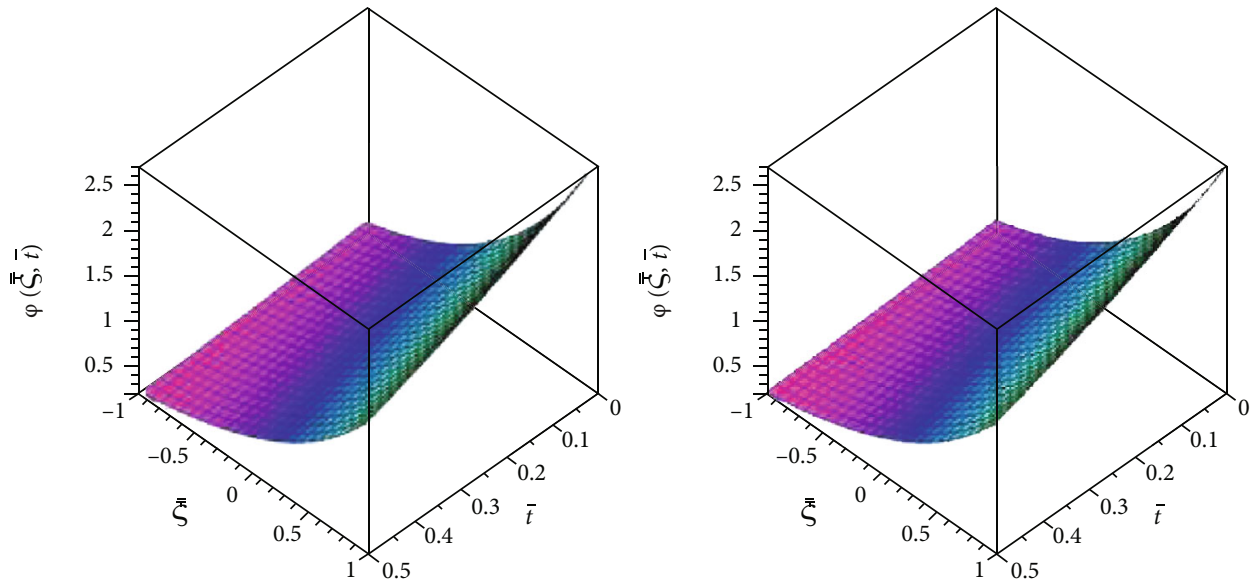

FIgURE 5: The exact and analytical solution figures of Example 2 at $\gamma=1$.

The exact result is

$$
\varphi(\bar{\xi}, \bar{t})=e^{\bar{\xi}-2 \bar{t}}
$$

In Figure 1, the first and second graphs show the exact and NDM solutions of $\varphi(\bar{\xi}, \bar{t})$ for Example 1 at $\gamma=1$. Figure 2 shows the different fractional-order graphs of $\varphi(\bar{\xi}$, $\bar{t})$ at $\gamma=0.8$ and $\gamma=0.6$, and Figure 3 shows the fractionalorder graph at $\gamma=0.40$. The graphs show the close relation between the exact and actual solutions with each other. Figure 4 shows the two- and three-dimensional graphs of different fractional-order $\gamma$.

Example 2. Consider the fractional-order telegraph equation

$$
\frac{\partial^{2 \gamma} \varphi}{\partial \bar{\xi}^{2 \gamma}}=\frac{\partial^{2} \varphi}{\partial \bar{t}^{2}}+\frac{\partial \varphi}{\partial \bar{t}}+\varphi, \quad 0<\gamma \leq 1, \bar{t} \geq 0
$$

with the initial condition

$$
\begin{aligned}
\varphi(0, \bar{t}) & =e^{-\bar{t}}, \\
\varphi_{\bar{\xi}}(0, \bar{t}) & =e^{-\bar{t}}
\end{aligned}
$$

Now, applying the natural transformation to (37), we get

$$
\mathcal{N}\left[\frac{\partial^{2 \gamma} \varphi}{\partial \bar{\xi}^{2 \gamma}}\right]=\mathcal{N}\left[\frac{\partial^{2} \varphi}{\partial \bar{t}^{2}}+\frac{\partial \varphi}{\partial \bar{t}}+\varphi\right]
$$

Using the inverse natural transformation,

$$
\begin{aligned}
\varphi(\bar{\xi}, \bar{t})= & \mathscr{N}^{-1}\left[\frac{\varphi(0, \bar{t})}{s}+\frac{\mu}{s^{2}} \varphi_{\bar{\xi}}(0, \bar{t})+\frac{(\mu / s)+\gamma(1-(\mu / s))}{(\mu / s)^{2}} \mathcal{N}\right. \\
& \left.\cdot\left[\frac{\partial^{2} \varphi}{\partial \bar{t}^{2}}+\frac{\partial \varphi}{\partial \bar{t}}+\varphi\right]\right] .
\end{aligned}
$$



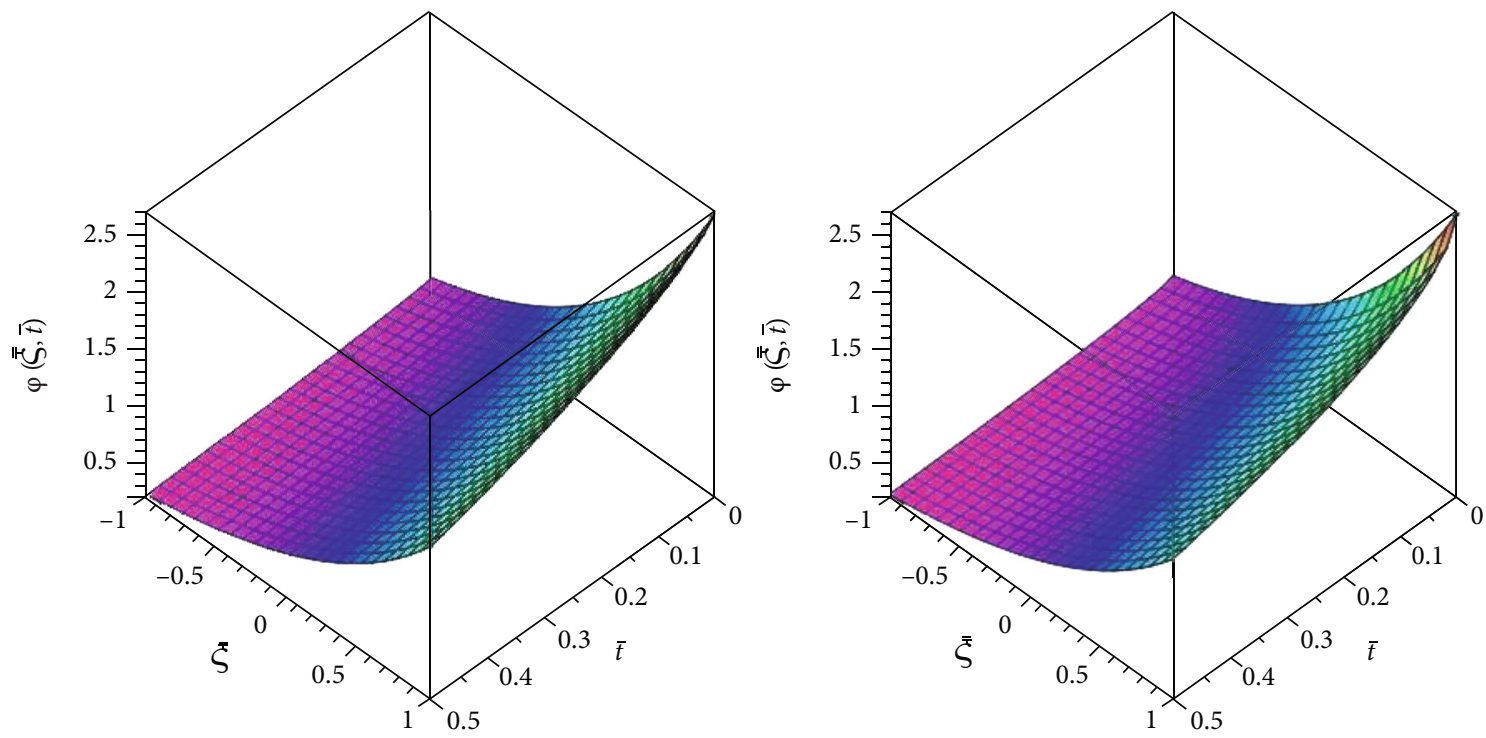

Figure 6: The different fractional-order graphs of $\varphi(\bar{\xi}, \bar{t})$ at $\gamma=0.8$ and $\gamma=0.6$.

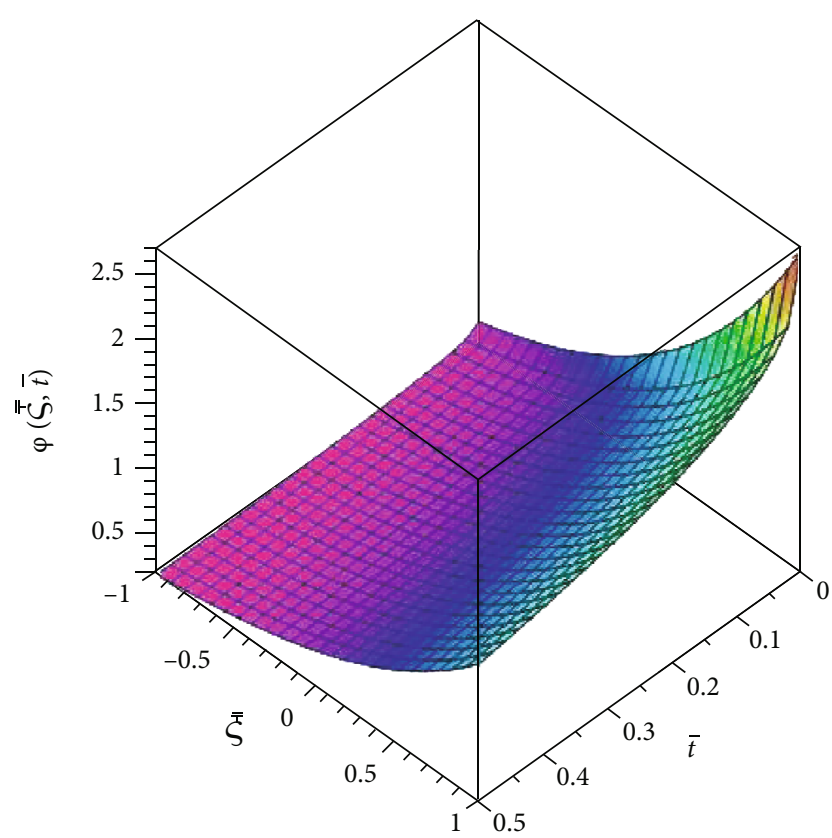

FIGURE 7: The fractional-order graph at $\gamma=0.40$.

Applying the Adomian decomposition method, we get

$$
\begin{gathered}
\varphi_{0}(\bar{\xi}, \bar{t})=\mathcal{N}^{-1}\left[\frac{\varphi(0, \bar{t})}{s}+\frac{\varphi_{\bar{\xi}}(0, \bar{t})}{s^{2}}\right]=\mathcal{N}^{-1}\left[\frac{e^{-\bar{t}}}{s}+\frac{\mu e^{-\bar{t}}}{s^{2}}\right], \\
\varphi_{0}(\bar{\xi}, \bar{t})=e^{-\bar{t}}(1+\bar{\xi}), \\
\varphi_{j+1}(\bar{\xi}, \bar{t})=\mathcal{N}^{-1}\left[\frac{(\mu / s)+\gamma(1-(\mu / s))}{(\mu / s)^{2}} \mathcal{N}\left[\frac{\partial^{2} \varphi_{j}}{\partial \bar{t}^{2}}+\frac{\partial \varphi_{j}}{\partial \bar{t}}+\varphi_{j}\right]\right], \quad j=0,1,2, \cdots .
\end{gathered}
$$

For $j=0$,

$$
\begin{aligned}
\varphi_{1}(\bar{\xi}, \bar{t}) & =\mathcal{N}^{-1}\left[\frac{(\mu / s)+\gamma(1-(\mu / s))}{(\mu / s)^{2}} \mathcal{N}\left[\frac{\partial^{2} \varphi_{0}}{\partial \bar{t}^{2}}+\frac{\partial \varphi_{0}}{\partial \bar{t}}+\varphi_{0}\right]\right] \\
& =e^{-\bar{t}}\left(1-\gamma+\bar{\xi}+\frac{\gamma \bar{\xi}^{2}}{2}\right) .
\end{aligned}
$$

The subsequent terms are

$$
\begin{aligned}
\varphi_{2}(\bar{\xi}, \bar{t}) & =\mathcal{N}^{-1}\left[\frac{(\mu / s)+\gamma(1-(\mu / s))}{(\mu / s)^{2}} \mathcal{N}\left[\frac{\partial^{2} \varphi_{1}}{\partial \bar{t}^{2}}+\frac{\partial \varphi_{1}}{\partial \bar{t}}+\varphi_{1}\right]\right] \\
& =e^{-\bar{t}}\left(\bar{\xi}-\gamma \bar{\xi}+\frac{\bar{\xi}^{2}}{2}+\frac{\gamma}{6} \bar{\xi}^{3}\right), \\
\varphi_{3}(\bar{\xi}, t) & =\mathscr{N}^{-1}\left[\frac{(\mu / s)+\gamma(1-(\mu / s))}{(\mu / s)^{2}} \mathcal{N}\left[\frac{\partial^{2} \varphi_{2}}{\partial \bar{t}^{2}}+\frac{\partial \varphi_{2}}{\partial \bar{t}}+\varphi_{2}\right]\right] \\
& =e^{-\bar{t}}\left(\frac{\bar{\xi}^{2}}{2}-\frac{\gamma \bar{\xi}^{2}}{2}+\frac{\gamma \bar{\xi}^{4}}{24}+\frac{\bar{\xi}^{3}}{6}\right),
\end{aligned}
$$

The NDM solution for Example 2 is

$$
\varphi(\bar{\xi}, \bar{t})=\varphi_{0}(\bar{\xi}, \bar{t})+\varphi_{1}(\bar{\xi}, \bar{t})+\varphi_{2}(\bar{\xi}, \bar{t})+\varphi_{3}(\bar{\xi}, \bar{t})+\varphi_{4}(\bar{\xi}, \bar{t}) \cdots
$$



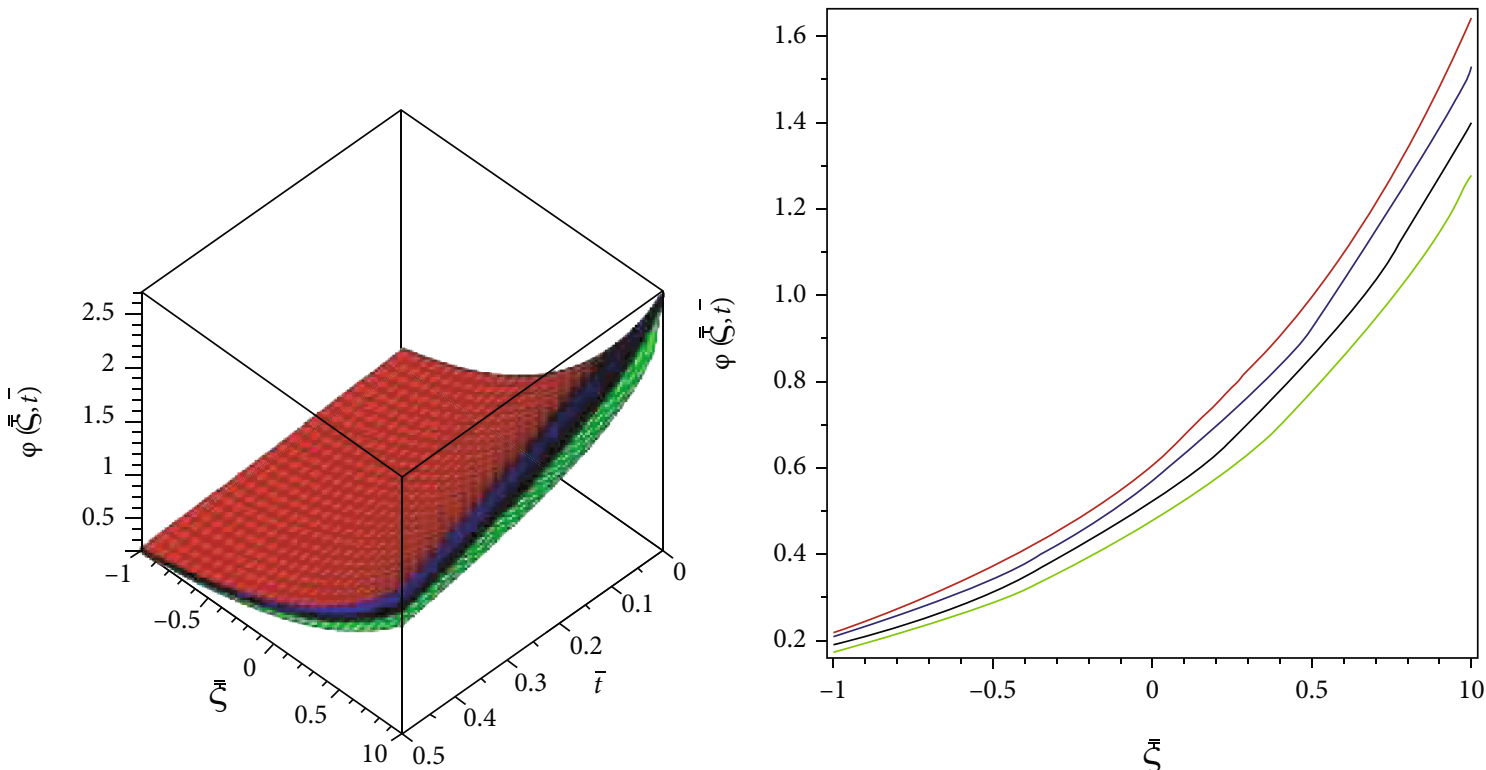

Figure 8: The 3D and 2D different fractional-order graphs of $\gamma$.
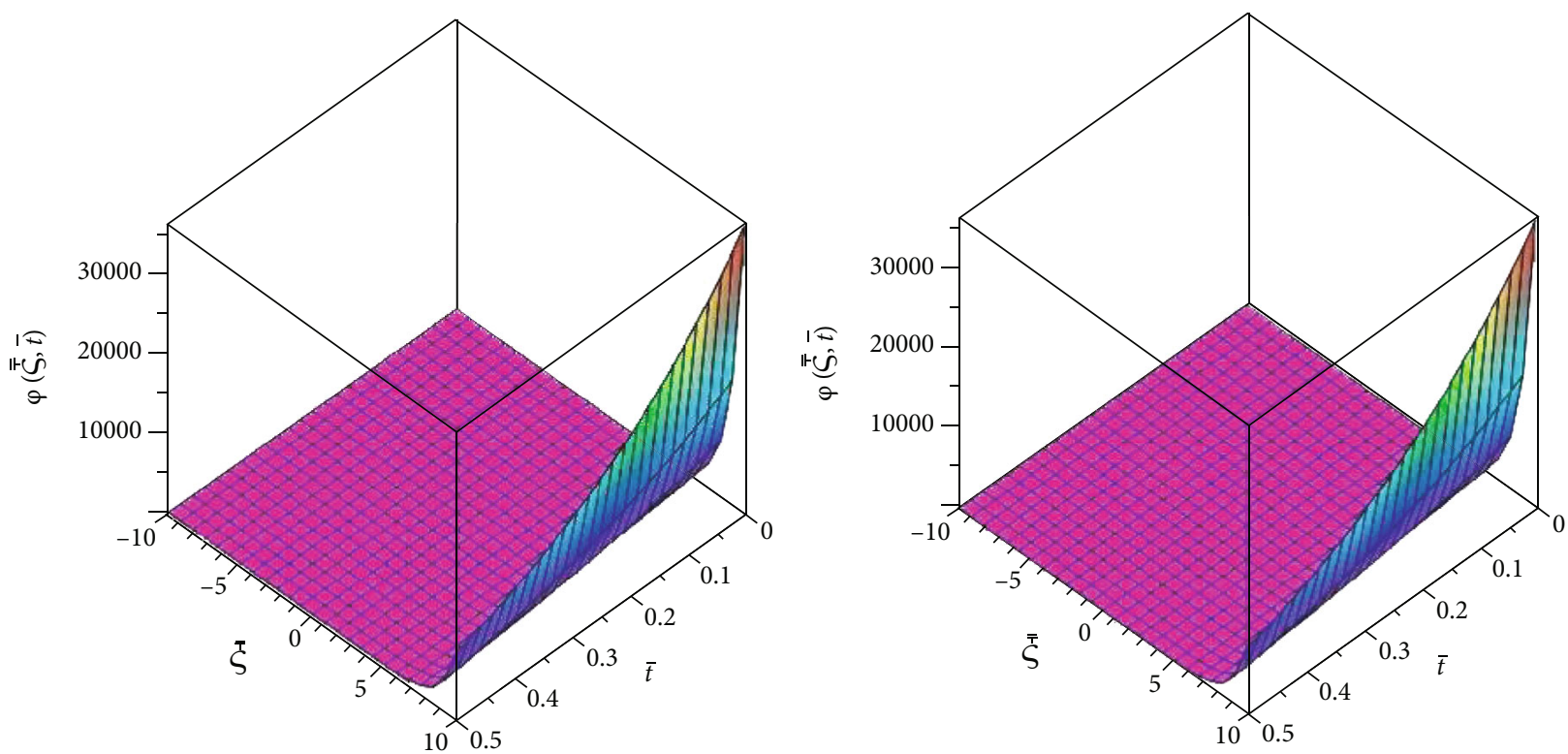

FIgURE 9: The exact and analytical solution figures of Example 3 at $\gamma=1$.

$$
\begin{aligned}
\varphi(\bar{\xi}, \bar{t})= & e^{-\bar{t}}\left[2+3 \bar{\xi}-\gamma+\frac{\gamma \bar{\xi}^{2}}{2}-\gamma \bar{\xi}+\frac{\bar{\xi}^{2}}{2}+\frac{\gamma}{6} \bar{\xi}^{3}+\frac{\bar{\xi}^{2}}{2}-\frac{\gamma \bar{\xi}^{2}}{2}\right. \\
& \left.+\frac{\gamma \bar{\xi}^{4}}{24}+\frac{\bar{\xi}^{3}}{6}+\cdots\right] .
\end{aligned}
$$

The exact result is

$$
\varphi(\bar{\xi}, \bar{t})=e^{\bar{\xi}-\bar{t}}
$$

In Figure 5, the first and second graphs show the exact and NDM solutions of $\varphi(\bar{\xi}, \bar{t})$ for Example 2 at $\gamma=1$. Figure 6 shows the different fractional-order graphs of $\varphi(\bar{\xi}, \bar{t})$ at $\gamma=0.8$ and $\gamma$ $=0.6$, and Figure 7 shows the fractional-order graph at $\gamma=$ 0.40 . The graphs show the close relation between the exact and actual solutions with each other. Figure 8 shows the twoand three-dimensional graphs of different fractional-order $\gamma$.

Example 3. Consider the fractional linear telegraph equation

$$
\frac{\partial^{2 \gamma} \varphi}{\partial \bar{t}^{2 \gamma}}+3 \frac{\partial^{\gamma} \varphi}{\partial \bar{t}^{\gamma}}+2 \varphi=\frac{\partial^{2} \varphi}{\partial \bar{\xi}^{2}}+\frac{\partial^{2} \varphi}{\partial \bar{\zeta}^{2}}, \quad 0<\gamma \leq 1, t \geq 0
$$



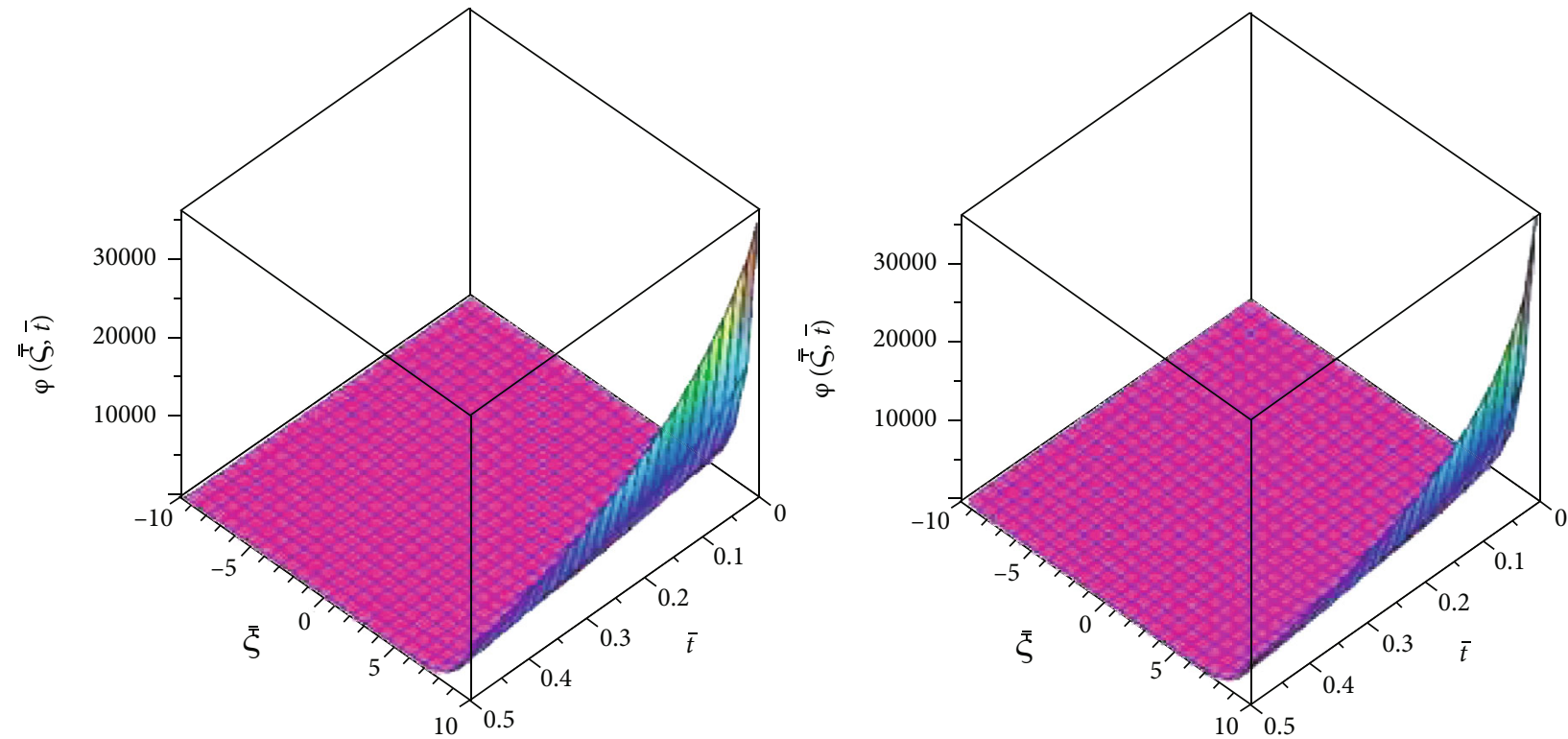

Figure 10: The different fractional-order graphs of $\varphi(\bar{\xi}, \bar{t})$ at $\gamma=0.8$ and $\gamma=0.6$.

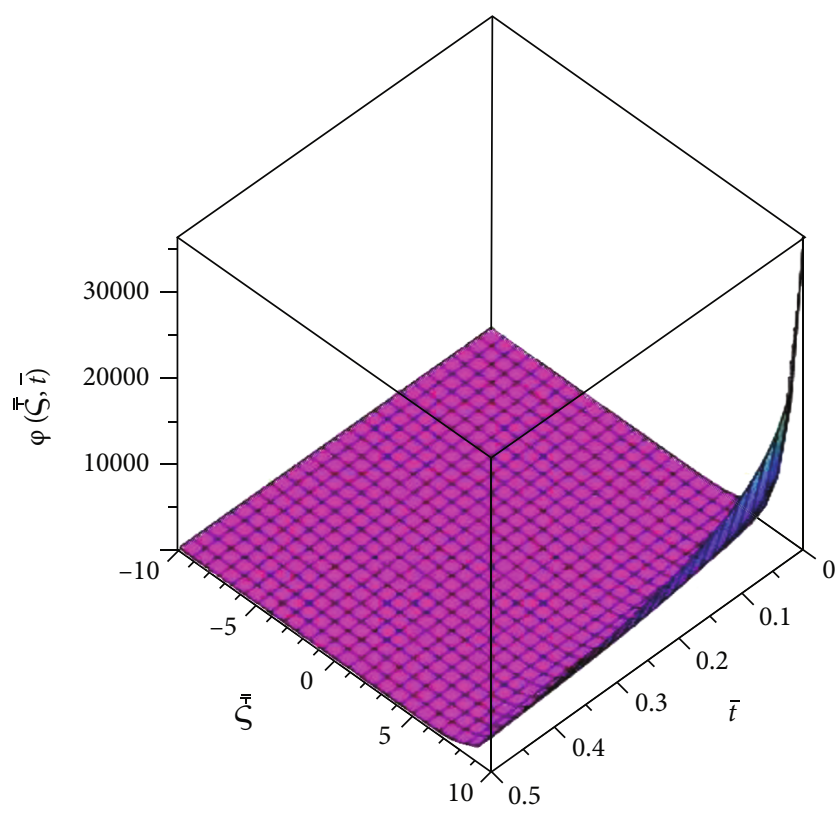

Figure 11: The fractional-order graph at $\gamma=0.40$.

with the initial condition

$$
\begin{gathered}
\varphi(\bar{\xi}, \bar{\zeta}, 0)=e^{\bar{\xi}+\bar{\zeta}} \\
\varphi_{\bar{t}}(\bar{\xi}, \bar{\zeta}, 0)=-3 e^{\bar{\xi}+\bar{\zeta}} .
\end{gathered}
$$

Using the natural transformation to equation (46), we get

$$
\mathcal{N}\left[\frac{\partial^{2 \gamma} \varphi}{\partial \bar{t}^{2 \gamma}}\right]=-\mathcal{N}\left[3 \frac{\partial^{\gamma} \varphi}{\partial \bar{t}^{\gamma}}+2 \varphi-\frac{\partial^{2} \varphi}{\partial \bar{\xi}^{2}}-\frac{\partial^{2} \varphi}{\partial \bar{\zeta}^{2}}\right] .
$$

Applying the inverse natural transformation,

$$
\begin{aligned}
\varphi(\bar{\xi}, \bar{\zeta}, \bar{t})= & \mathscr{N}^{-1}\left[\frac{\varphi(\bar{\xi}, \bar{\zeta}, 0)}{s}+\frac{\varphi_{\bar{t}}(\bar{\xi}, \bar{\zeta}, 0)}{s^{2}}-\frac{(\mu / s)+\gamma(1-(\mu / s))}{(\mu / s)^{2}} \mathcal{N}\right. \\
& \left.\cdot\left[3 \frac{\partial^{\gamma} \varphi}{\partial \bar{t}^{\gamma}}+2 \varphi-\frac{\partial^{2} \varphi}{\partial \bar{\xi}^{2}}-\frac{\partial^{2} \varphi}{\partial \bar{\zeta}^{2}}\right]\right] .
\end{aligned}
$$

Implementing the Adomian decomposition method, we get

$$
\begin{aligned}
\varphi_{0}(\bar{\xi}, \bar{\zeta}, \bar{t}) & =\mathscr{N}^{-1}\left[\frac{\varphi(\bar{\xi}, \bar{\zeta}, 0)}{s}+\frac{\mu}{s^{2}} \varphi_{\bar{t}}(\bar{\xi}, \bar{\zeta}, 0)\right] \\
& =\mathscr{N}^{-1}\left[\frac{e^{\bar{\xi}+\bar{\zeta}}}{s}-\frac{3 \mu e^{\bar{\xi}+\bar{\zeta}}}{s^{2}}\right]
\end{aligned}
$$

$$
\varphi_{0}(\bar{\xi}, \bar{\zeta}, \bar{t})=e^{\bar{\xi}+\bar{\zeta}}(1-3 \bar{t})
$$

$$
\begin{aligned}
\varphi_{j+1}(\bar{\xi}, \bar{\zeta}, \bar{t})= & -\mathscr{N}^{-1}\left[\frac{(\mu / s)+\gamma(1-(\mu / s))}{(\mu / s)^{2}} \mathcal{N}\right. \\
& \left.\cdot\left[3 \frac{\partial^{\gamma} \varphi_{j}}{\partial \bar{t}^{\gamma}}+2 \varphi_{j}-\frac{\partial^{2} \varphi_{j}}{\partial \bar{\xi}^{2}}-\frac{\partial^{2} \varphi_{j}}{\partial \bar{\zeta}^{2}}\right]\right], \quad j=0,1,2, \cdots
\end{aligned}
$$



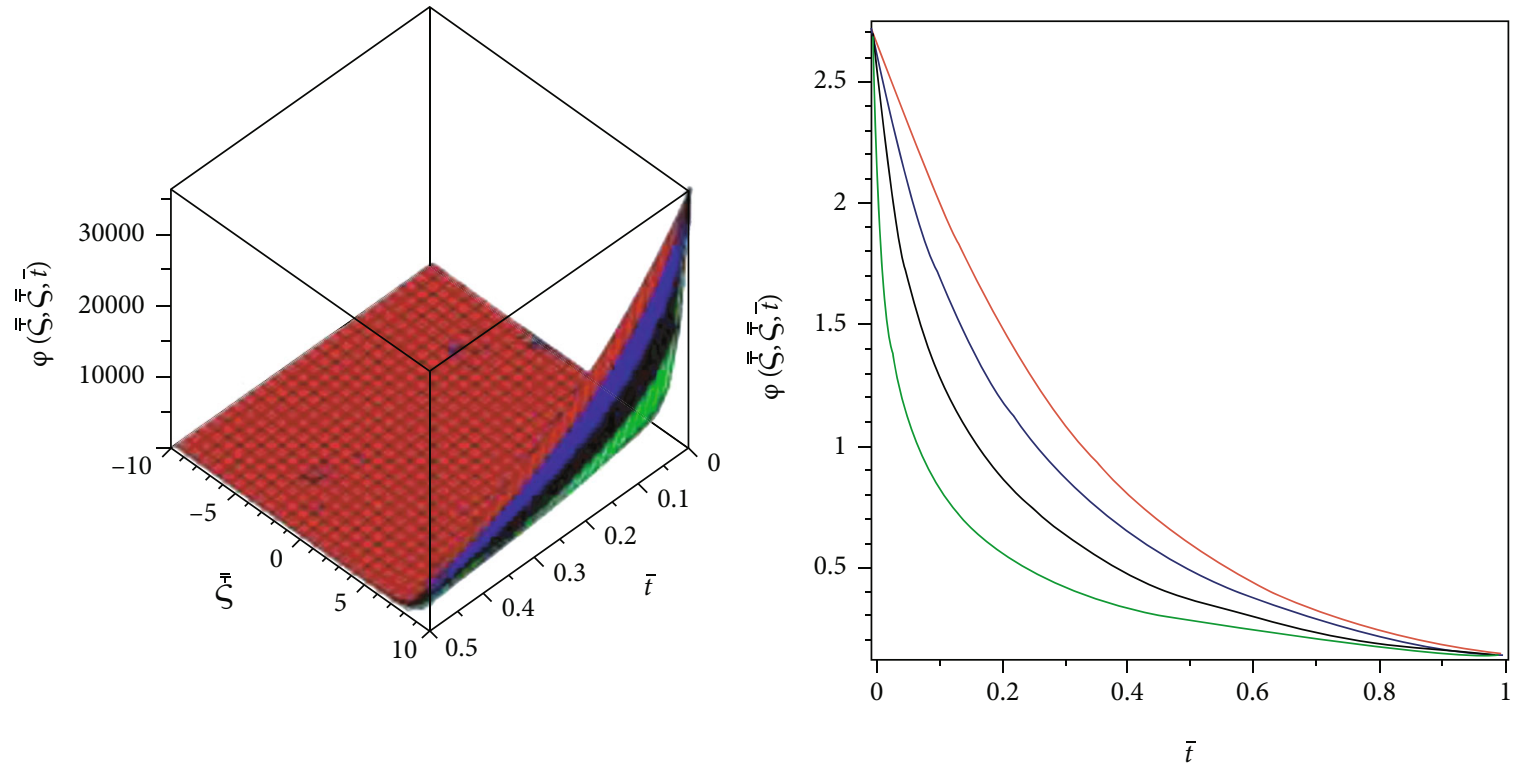

Figure 12: The 3D and 2D different fractional-order graphs of $\gamma$.

For $j=0$,

$$
\begin{gathered}
\varphi_{1}(\bar{\xi}, \bar{\zeta}, \bar{t})=-\mathcal{N}^{-1}\left[\frac{(\mu / s)+\gamma(1-(\mu / s))}{(\mu / s)^{2}} \mathcal{N}\left[3 \frac{\partial^{\gamma} \varphi_{0}}{\partial \bar{t}^{\gamma}}+2 \varphi_{0}-\frac{\partial^{2} \varphi_{0}}{\partial \bar{\xi}^{2}}-\frac{\partial^{2} \varphi_{0}}{\partial \bar{\zeta}^{2}}\right]\right], \\
\varphi_{1}(\bar{\xi}, \bar{\zeta}, \bar{t})=9 e^{\bar{\xi}+\bar{\zeta}}\left(\bar{t}-\gamma \bar{t}+\frac{\gamma \bar{t}^{2}}{2}\right) .
\end{gathered}
$$

The subsequent terms are

$$
\begin{aligned}
\varphi_{2}(\bar{\xi}, \bar{\zeta}, \bar{t}) & =-\mathcal{N}^{-1}\left[\frac{(\mu / s)+\gamma(1-(\mu / s))}{(\mu / s)^{2}} \mathcal{N}\left[3 \frac{\partial^{\gamma} \varphi_{1}}{\partial \bar{t}^{\prime}}+2 \varphi_{1}-\frac{\partial^{2} \varphi_{1}}{\partial \bar{\xi}^{2}}-\frac{\partial^{2} \varphi_{1}}{\partial \bar{\zeta}^{2}}\right]\right] \\
& =-27 e^{\bar{\xi}+\bar{\zeta}}\left(\frac{\bar{t}^{2}}{2}-\frac{\gamma \bar{t}^{2}}{2}+\frac{\gamma \bar{t}^{3}}{6}\right), \\
\varphi_{3}(\bar{\xi}, \bar{\zeta}, \bar{t}) & =-\mathscr{N}^{-1}\left[\frac{(\mu / s)+\gamma(1-(\mu / s))}{(\mu / s)^{2}} \mathcal{N}\left[3 \frac{\partial^{\gamma} \varphi_{2}}{\partial \bar{t}^{\prime}}+2 \varphi_{2}-\frac{\partial^{2} \varphi_{2}}{\partial \bar{\xi}^{2}}-\frac{\partial^{2} \varphi_{2}}{\partial \bar{\zeta}^{2}}\right]\right] \\
& =81 e^{\bar{\xi}+\bar{\xi} \bar{\zeta}}\left(\frac{\bar{t}^{3}}{6}-\frac{\gamma \bar{t}^{3}}{6}+\frac{\gamma \bar{t}^{4}}{24}\right),
\end{aligned}
$$

The NDM result for Example 3 is

$$
\begin{aligned}
\varphi(\bar{\xi}, \bar{\zeta}, \bar{t})= & \varphi_{0}(\bar{\xi}, \bar{\zeta}, \bar{t})+\varphi_{1}(\bar{\xi}, \bar{\zeta}, \bar{t})+\varphi_{2}(\bar{\xi}, \bar{\zeta}, \bar{t})+\varphi_{3}(\bar{\xi}, \bar{\zeta}, \bar{t})+\varphi_{4}(\bar{\xi}, \bar{\zeta}, \bar{t}) \cdots \\
\varphi(\bar{\xi}, \bar{\zeta}, \bar{t})= & e^{\bar{\xi}+\bar{\zeta}}\left[1-3 \bar{t}+9\left(\bar{t}-\gamma \bar{t}+\frac{\gamma \bar{t}^{2}}{2}\right)-27\left(\frac{\bar{t}^{2}}{2}-\frac{\gamma \bar{t}^{2}}{2}+\frac{\gamma \bar{t}^{3}}{6}\right)\right. \\
& \left.+81\left(\frac{\bar{t}^{3}}{6}-\frac{\gamma \bar{t}^{3}}{6}+\frac{\gamma \bar{t}^{4}}{24}\right) \cdots\right] .
\end{aligned}
$$

When $\gamma=1$, then the NDM result is

$$
\varphi(\bar{\xi}, \bar{\zeta}, \bar{t})=e^{\bar{\xi}+\bar{\zeta}}\left[1-3 \bar{t}+\frac{(3 \bar{t})^{2}}{2 !}-\frac{(3 \bar{t})^{3}}{3 !}+\frac{(3 \bar{t})^{4}}{4 !} \cdots\right] .
$$

The exact result is

$$
\varphi(\bar{\xi}, \bar{\zeta}, \bar{t})=e^{\bar{\xi}+\bar{\zeta}-3 \bar{t}}
$$

In Figure 9, the first and second graphs show the exact and NDM solutions of $\varphi(\bar{\xi}, \bar{t})$ for Example 3 at $\gamma=1$. Figure 10 shows the different fractional-order graphs of $\varphi(\bar{\xi}$ $, \bar{t})$ at $\gamma=0.8$ and $\gamma=0.6$, and Figure 11 shows the fractional-order graph at $\gamma=0.40$. The graphs show the close relation between the exact and actual solutions with each other. Figure 12 shows the two- and three-dimensional graphs of different fractional-order $\gamma$.

Example 4. Consider the fractional linear telegraph equation

$$
\frac{\partial^{2 \gamma} \varphi}{\partial \bar{t}^{2 \gamma}}+2 \frac{\partial^{\gamma} \varphi}{\partial \bar{t}^{\gamma}}+3 \varphi=\frac{\partial^{2} \varphi}{\partial \bar{\xi}^{2}}+\frac{\partial^{2} \varphi}{\partial \bar{\zeta}^{2}}+\frac{\partial^{2} \varphi}{\partial \chi^{2}}, \quad 0<\gamma \leq 1, \bar{t} \geq 0,
$$

with the initial condition

$$
\begin{aligned}
\varphi(\bar{\xi}, \bar{\zeta}, \chi, 0) & =\sinh (\bar{\xi}) \sinh (\bar{\zeta}) \sinh (\chi) \\
\varphi_{\bar{t}}(\bar{\xi}, \bar{\zeta}, \chi, 0) & =-\sinh (\bar{\xi}) \sinh (\bar{\zeta}) \sinh (\chi)
\end{aligned}
$$



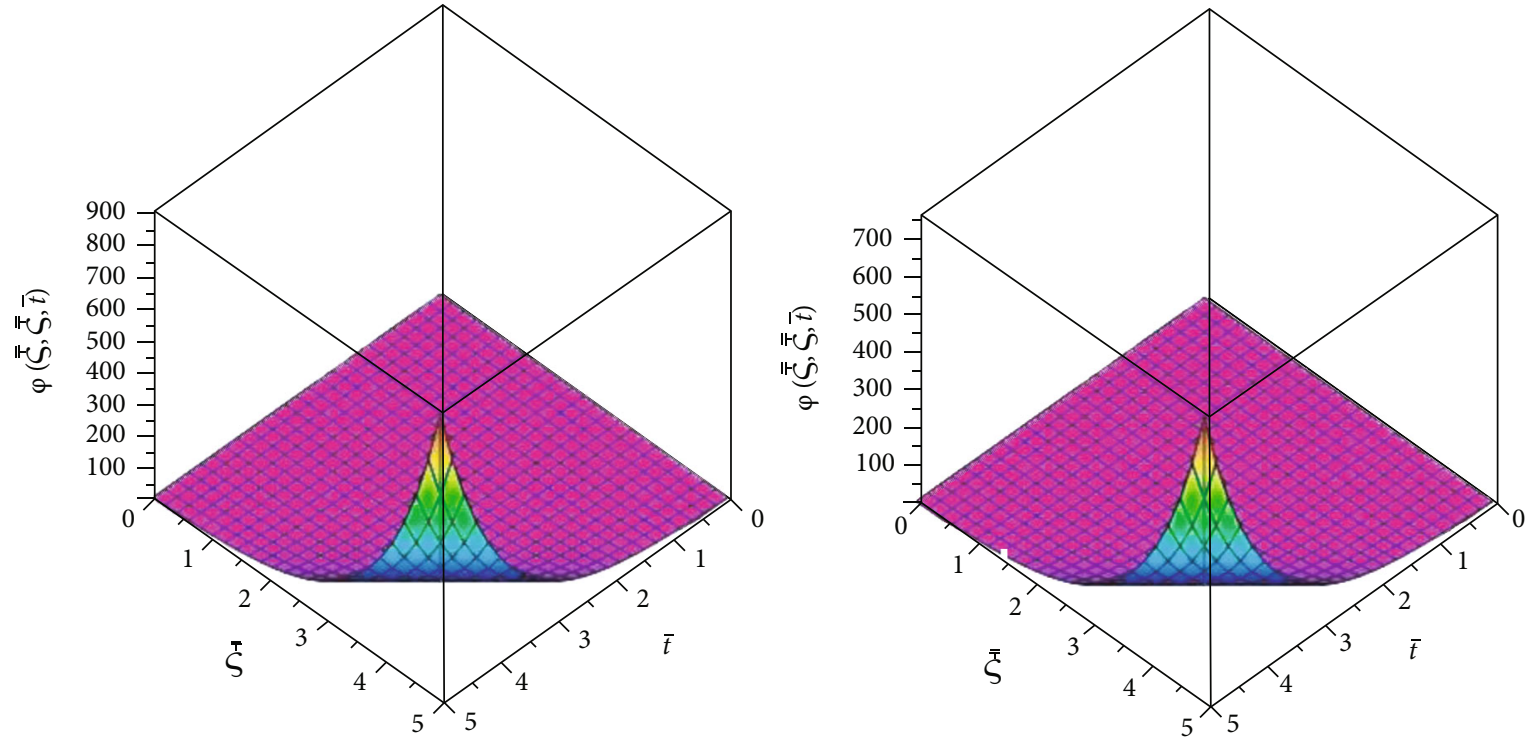

FIGURE 13: The exact and analytical solution figures of Example 4 at $\gamma=1$.

Taking the natural transformation of equation (56),

$\mathscr{N}\left[\frac{\partial^{2 \gamma} \varphi}{\partial \bar{t}^{2 \gamma}}\right]=-\mathcal{N}\left[2 \frac{\partial^{\gamma} \varphi}{\partial \bar{t}^{\gamma}}+3 \varphi-\frac{\partial^{2} \varphi}{\partial \bar{\xi}^{2}}-\frac{\partial^{2} \varphi}{\partial \bar{\zeta}^{2}}-\frac{\partial^{2} \varphi}{\partial \chi^{2}}\right]$.

$$
\begin{aligned}
\varphi_{j+1}(\bar{\xi}, \bar{\zeta}, \chi, \bar{t})= & -\mathcal{N}^{-1}\left[\frac{(\mu / s)+\gamma(1-(\mu / s))}{(\mu / s)^{2}} \mathscr{N}\right. \\
& \left.\cdot\left[2 \frac{\partial^{\gamma} \varphi_{j}}{\partial \bar{t}^{\gamma}}+3 \varphi_{j}-\frac{\partial^{2} \varphi_{j}}{\partial \bar{\xi}^{2}}-\frac{\partial^{2} \varphi_{j}}{\partial \bar{\zeta}^{2}}-\frac{\partial^{2} \varphi_{j}}{\partial \chi^{2}}\right]\right], \quad j=0,1,2, \cdots
\end{aligned}
$$

Using the inverse natural transformation,

$$
\begin{aligned}
\varphi(\bar{\xi}, \bar{\zeta}, \chi, \bar{t})= & \mathscr{N}^{-1}\left[\frac{\varphi(\bar{\xi}, \bar{\zeta}, \chi, 0)}{s}+\frac{\mu}{s^{2}} \varphi_{\bar{t}}(\bar{\xi}, \bar{\zeta}, \chi, 0)\right] \\
& -\mathcal{N}^{-1}\left[\frac{(\mu / s)+\gamma(1-(\mu / s))}{(\mu / s)^{2}} \mathscr{N}\right. \\
& \left.\cdot\left[2 \frac{\partial^{\gamma} \varphi}{\partial \bar{t}^{\gamma}}+3 \varphi-\frac{\partial^{2} \varphi}{\partial \bar{\xi}^{2}}-\frac{\partial^{2} \varphi}{\partial \bar{\zeta}^{2}}-\frac{\partial^{2} \varphi}{\partial \chi^{2}}\right]\right] .
\end{aligned}
$$

Applying the Adomian decomposition method, we get

$$
\begin{aligned}
\varphi_{0}(\bar{\xi}, \bar{\zeta}, \chi, \bar{t})= & \mathscr{N}^{-1}\left[\frac{\varphi(\bar{\xi}, \bar{\zeta}, \chi, 0)}{s}+\frac{\varphi_{\bar{t}}(\bar{\xi}, \bar{\zeta}, \chi, 0)}{s^{2}}\right] \\
= & \mathscr{N}^{-1}\left[\frac{\sinh (\bar{\xi}) \sinh (\bar{\zeta}) \sinh (\chi)}{s}\right. \\
& \left.-\frac{\sinh (\bar{\xi}) \sinh (\bar{\zeta}) \sinh (\chi)}{s^{2}}\right],
\end{aligned}
$$$$
\varphi_{0}(\bar{\xi}, \bar{\zeta}, \chi, \bar{t})=\sinh (\bar{\xi}) \sinh (\bar{\zeta}) \sinh (\chi)(1-\bar{t})
$$

For $j=0$,

$$
\begin{aligned}
\varphi_{1}(\bar{\xi}, \bar{\zeta}, \chi, \bar{t})= & -\mathcal{N}^{-1}\left[\frac { ( \mu / s ) + \gamma ( 1 - ( \mu / s ) ) } { ( \mu / s ) ^ { 2 } } \mathcal { N } \left[2 \frac{\partial^{\gamma} \varphi_{0}}{\partial \bar{t}^{\gamma}}+3 \varphi_{0}\right.\right. \\
& \left.\left.-\frac{\partial^{2} \varphi_{0}}{\partial \bar{\xi}^{2}}-\frac{\partial^{2} \varphi_{0}}{\partial \bar{\zeta}^{2}}-\frac{\partial^{2} \varphi_{0}}{\partial \chi^{2}}\right]\right] \\
\varphi_{1}(\bar{\xi}, \bar{\zeta}, \chi, \bar{t})= & -\mathscr{N}^{-1}\left[\frac{2 \sinh (\bar{\xi}) \sinh (\bar{\zeta}) \sinh (\chi)}{s^{\gamma+2}}\right],
\end{aligned}
$$

$\varphi_{1}(\bar{\xi}, \bar{\zeta}, \chi, \bar{t})=-2 \sinh (\bar{\xi}) \sinh (\bar{\zeta}) \sinh (\chi)\left(\bar{t}-\gamma \bar{t}+\frac{\gamma \bar{t}^{2}}{2}\right)$

The subsequent terms are

$$
\begin{aligned}
\varphi_{2}(\bar{\xi}, \bar{\zeta}, \chi, \bar{t})= & -\mathcal{N}^{-1}\left[\frac { ( \mu / s ) + \gamma ( 1 - ( \mu / s ) ) } { ( \mu / s ) ^ { 2 } } \mathcal { N } \left[2 \frac{\partial^{\gamma} \varphi_{1}}{\partial \bar{t}^{\gamma}}+3 \varphi_{1}\right.\right. \\
& \left.\left.-\frac{\partial^{2} \varphi_{1}}{\partial \bar{\xi}^{2}}-\frac{\partial^{2} \varphi_{1}}{\partial \bar{\zeta}^{2}}-\frac{\partial^{2} \varphi_{1}}{\partial \chi^{2}}\right]\right], \\
\varphi_{2}(\bar{\xi}, \bar{\zeta}, \chi, \bar{t})= & 4 \sinh (\bar{\xi}) \sinh (\bar{\zeta}) \sinh (\chi)\left(\frac{\bar{t}^{2}}{2}-\frac{\gamma \bar{t}^{2}}{2}+\frac{\gamma \bar{t}^{3}}{6}\right),
\end{aligned}
$$



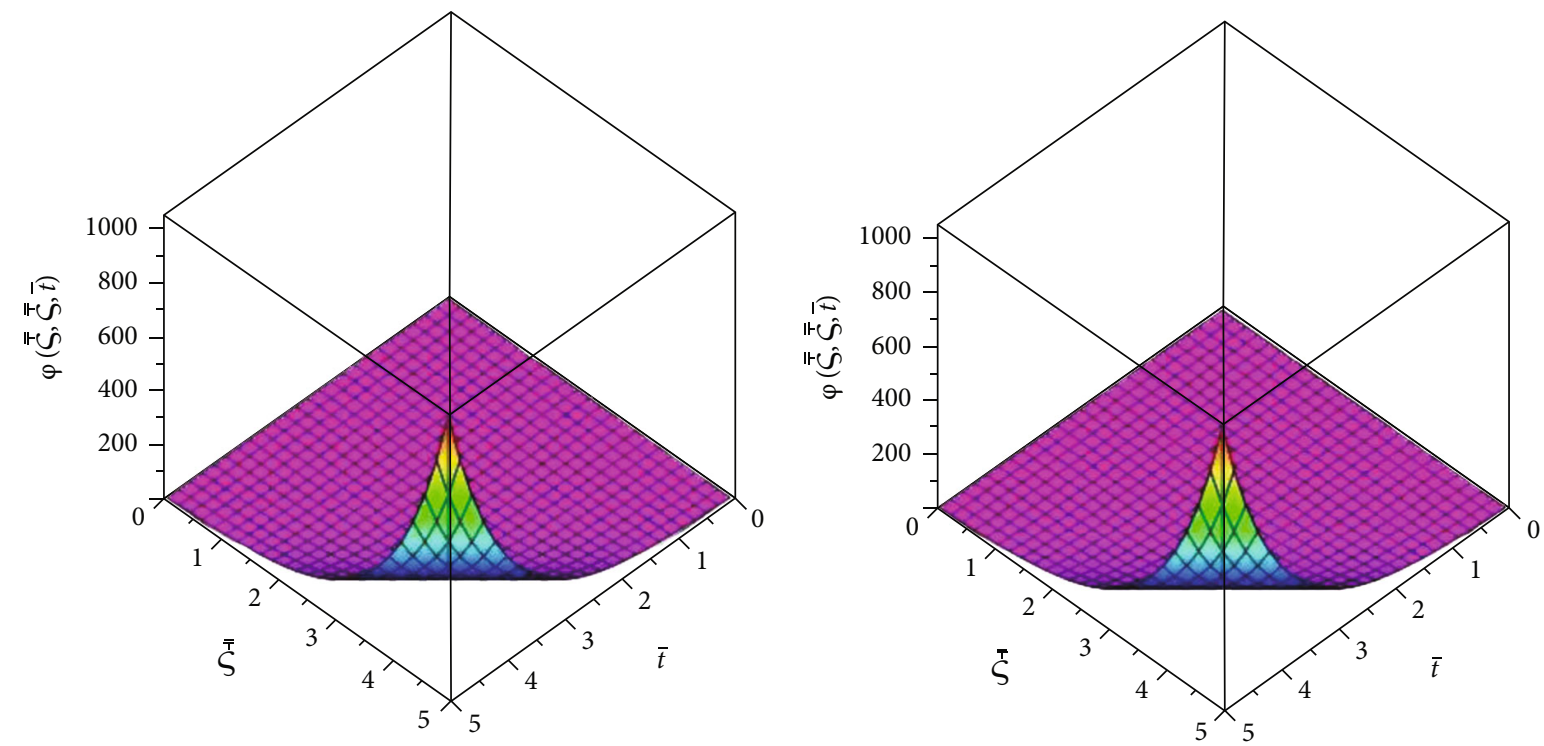

Figure 14: The different fractional-order graphs of $\varphi(\bar{\xi}, \bar{t})$ at $\gamma=0.8$ and $\gamma=0.6$.

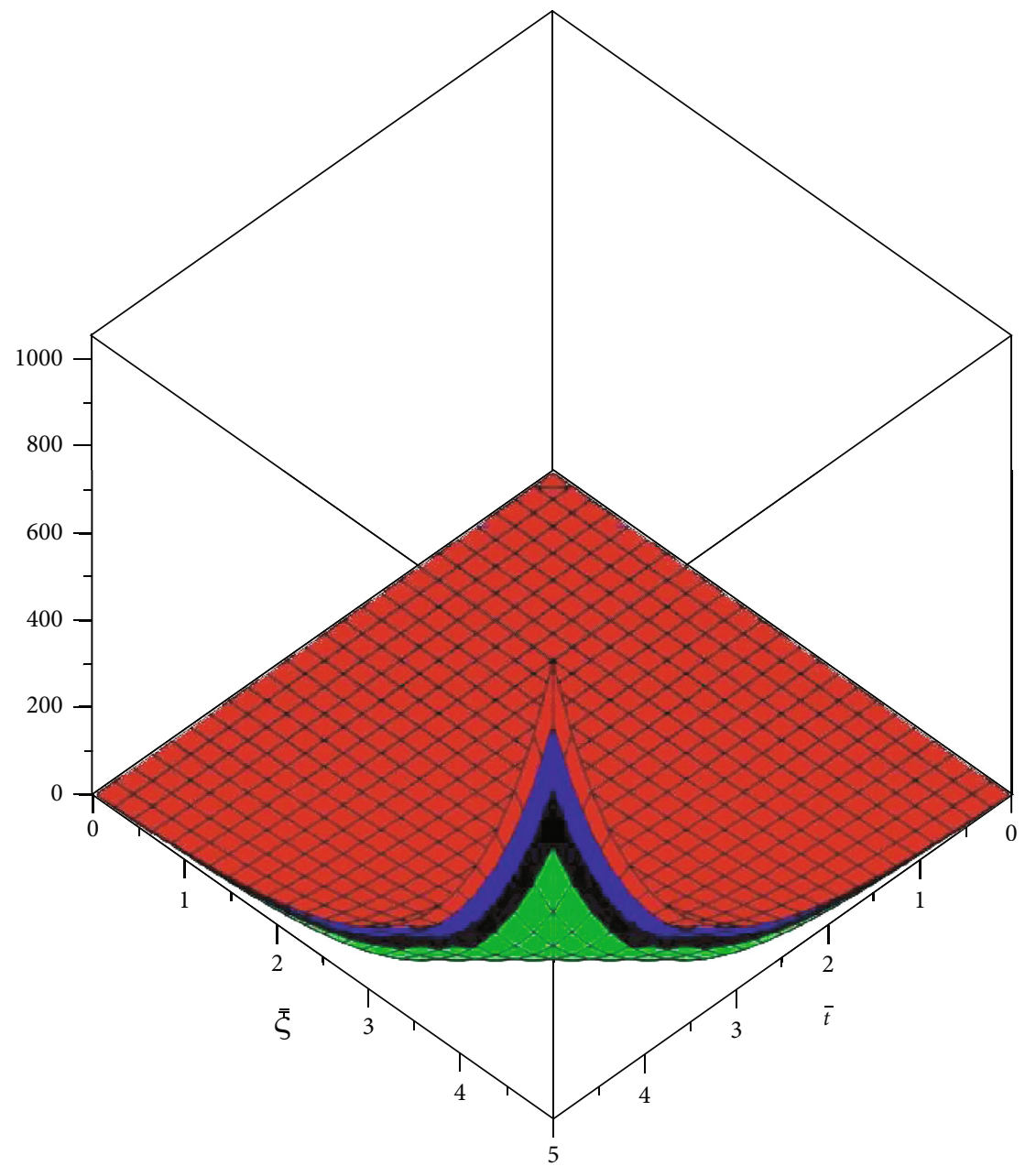

Figure 15: The 3D different fractional-order graphs of $\gamma$. 


$$
\begin{aligned}
\varphi_{3}(\bar{\xi}, \bar{\zeta}, \chi, \bar{t})= & -\mathcal{N}^{-1}\left[\frac { ( \mu / s ) + \gamma ( 1 - ( \mu / s ) ) } { ( \mu / s ) ^ { 2 } } \mathcal { N } \left[2 \frac{\partial^{\gamma} \varphi_{2}}{\partial \bar{t}^{\gamma}}+3 \varphi_{2}\right.\right. \\
& \left.\left.-\frac{\partial^{2} \varphi_{2}}{\partial \bar{\xi}^{2}}-\frac{\partial^{2} \varphi_{2}}{\partial \bar{\zeta}^{2}}-\frac{\partial^{2} \varphi_{2}}{\partial \chi^{2}}\right]\right], \\
\varphi_{3}(\bar{\xi}, \bar{\zeta}, \chi, \bar{t})= & -8 \sinh (\bar{\xi}) \sinh (\bar{\zeta}) \sinh (\chi)\left(\frac{\bar{t}^{3}}{6}-\frac{\gamma \bar{t}^{3}}{6}+\frac{\gamma \bar{t}^{4}}{24}\right),
\end{aligned}
$$

The NDM result for Example 4 is

$$
\begin{aligned}
& \varphi(\bar{\xi}, \bar{\zeta}, \chi, \bar{t})=\varphi_{0}(\bar{\xi}, \bar{\zeta}, \chi, \bar{t})+\varphi_{1}(\bar{\xi}, \bar{\zeta}, \chi, \bar{t})+\varphi_{2}(\bar{\xi}, \bar{\zeta}, \chi, \bar{t})+\varphi_{3}(\bar{\xi}, \bar{\zeta}, \chi, \bar{t})+\cdots \\
& \varphi(\bar{\xi}, \bar{\zeta}, \chi, \bar{t})= \sinh (\bar{\xi}) \sinh (\bar{\zeta}) \sinh (\chi)[1+\bar{t}-2 \\
& \cdot\left(t-\gamma \bar{t}+\frac{\gamma \bar{t}^{2}}{2}\right)+4\left(\frac{\bar{t}^{2}}{2}-\frac{\gamma \bar{t}^{2}}{2}+\frac{\gamma \bar{t}^{3}}{6}\right) \\
&\left.-8\left(\frac{\bar{t}^{3}}{6}-\frac{\gamma \bar{t}^{3}}{6}+\frac{\gamma \bar{t}^{4}}{24}\right)+\cdots\right]
\end{aligned}
$$

The exact result is

$$
\varphi(\bar{\xi}, \bar{\zeta}, \chi, \bar{t})=e^{-2 \bar{t}} \sinh (\bar{\xi}) \sinh (\bar{\zeta}) \sinh (\chi)
$$

In Figure 13, the first and second graphs show the exact and NDM solutions of $\varphi(\bar{\xi}, \bar{t})$ for Example 4 at $\gamma=1$. Figure 14 shows the different fractional-order graphs of $\varphi(\bar{\xi}$ $, \bar{t})$ at $\gamma=0.8$ and $\gamma=0.6$. The graphs show the close relation between the exact and actual solutions with each other. Figure 15 shows the three-dimensional graphs of different fractional-order $\gamma$.

\section{Conclusions}

In this article, we have investigated the telegraph equations through natural transformation with the Caputo-Fabrizio derivative. It is also shown that the fractional-order results were convergent to the actual result in the examples, as the fractional order approached the integer order. The implementation of the natural decomposition method in the illustrative problems has also confirmed that the fractional-order mathematical models can analyze any experimental data in a better manner compared to the integer-order models. Furthermore, by using different fractional orders, we could find a way to create appropriate mathematical models for any empirical data and thus understand practical implications. The natural decomposition method is simple in its principles; also, the natural decomposition method effectively solves linear and nonlinear fractional differential equations. It can be proved a promising technique for a large variety of such equations arising in mathematical physics. In the future, the natural transform decomposition technique modified with the help of different fractional operators such as AtanganaBaleanu and Yang-Abdel-Cattani operators is the most reliable method for solving different fractional-order linear and nonlinear partial differential equations.

\section{Data Availability}

The numerical data used to support the findings of this study are included within the article.

\section{Conflicts of Interest}

The authors declare that there are no conflicts of interest regarding the publication of this article.

\section{Acknowledgments}

This research was funded by the Deanship of Scientific Research at Princess Nourah Bint Abdulrahman University through the Fast-track Research Funding Program. The authors would like to thank the Deanship Scientific Research at Umm Al-Qura University for supporting this research work under Grant Code 19-SCI-1-01-004.

\section{References}

[1] V. K. Srivastava, M. K. Awasthi, R. K. Chaurasia, and M. Tamsir, "The telegraph equation and its solution by reduced differential transform method," Modelling and Simulation in Engineering, vol. 2013, 6 pages, 2013.

[2] V. K. Srivastava, M. K. Awasthi, and M. Tamsir, "RDTM solution of Caputo time fractional-order hyperbolic telegraph equation," AIP Advances, vol. 3, no. 3, article 032142, 2013.

[3] R. Jiwari, S. Pandit, and R. C. Mittal, "A differential quadrature algorithm for the numerical solution of the second-order one dimensional hyperbolic telegraph equation," International Journal of Nonlinear Science, vol. 13, pp. 259-266, 2012.

[4] M. Javidi and N. Nyamoradi, "Numerical solution of telegraph equation by using LT inversion technique," International journal of advanced mathematical sciences, vol. 1, pp. 64-77, 2013.

[5] M. Inc, A. Akgul, and A. Kiliman, "Explicit solution of telegraph equation based on reproducing kernel method," Journal of Function Spaces and Applications, vol. 2012, pp. 1-23, 2012.

[6] P. Veeresha and D. G. Prakasha, "Numerical solution for fractional model of telegraph equation by using q-HATM," 2018, https://arxiv.org/abs/1805.03968.

[7] H. Al-badrani, S. Saleh, H. O. Bakodah, and M. Al-Mazmumy, "Numerical solution for nonlinear telegraph equation by modified Adomian decomposition method," Nonlinear Analysis and Differential Equations, vol. 4, pp. 243-257, 2016.

[8] J. Biazar, H. Ebrahimi, and Z. Ayati, "An approximation to the solution of telegraph equation by variational iteration method," Numerical Methods for Partial Differential Equations, vol. 25, no. 4, pp. 797-801, 2009.

[9] M. Erfanian and M. Gachpazan, "A new method for solving of telegraph equation with Haar wavelet," International Journal of Mathematics and Computer Science, vol. 3, pp. 6-10, 2016.

[10] L. Galu, "Solution of some fractional order telegraph equations," Revista Colombiana de Matemáticas, vol. 48, pp. 247267, 2014. 
[11] Y. Wang and L. Mei, "Generalized finite difference/spectral Galerkin approximations for the time-fractional telegraph equation," Advances in Difference Equations, vol. 2017, no. 1, 2017.

[12] H. Latifizadeh, "The Sinc-collocation method for solving the telegraph equation," Journal of Computing and Information, vol. 1, pp. 13-17, 2013.

[13] M. Rawashdeh and S. Maitama, "Finding exact solutions of nonlinear PDEs using the natural decomposition method," Mathematicsl Methods in the Applied Sciences, vol. 40, no. 1, pp. 223-236, 2017.

[14] P. Veeresha, D. G. Prakasha, and H. M. Baskonus, "Novel simulations to the time-fractional Fishers equation," Mathematical Sciences, vol. 13, no. 1, pp. 33-42, 2019.

[15] D. G. Prakasha, P. Veeresha, and M. S. Rawashdeh, "Numerical solution for $(2+1)$-dimensional time-fractional coupled Burger equations using fractional natural decomposition method," Mathematicsl Methods in the Applied Sciences, vol. 42, no. 10, pp. 3409-3427, 2019. 